\title{
LHCb CP Violation at $\mathrm{LHCb}$ Early Results and Prospects

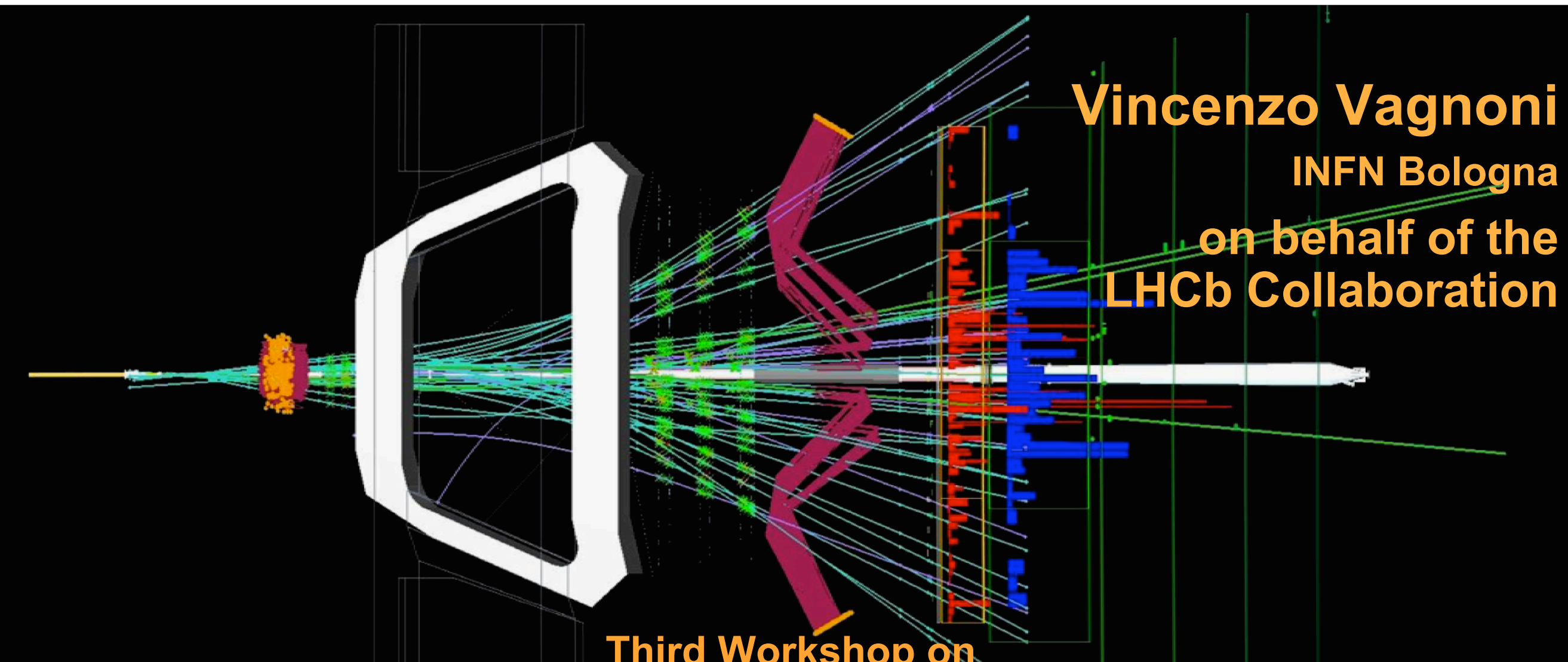

Theory, Phenomenology and Experiments in Neavy Flavour Physics 


\section{IHCP The LHCb Collaboration}

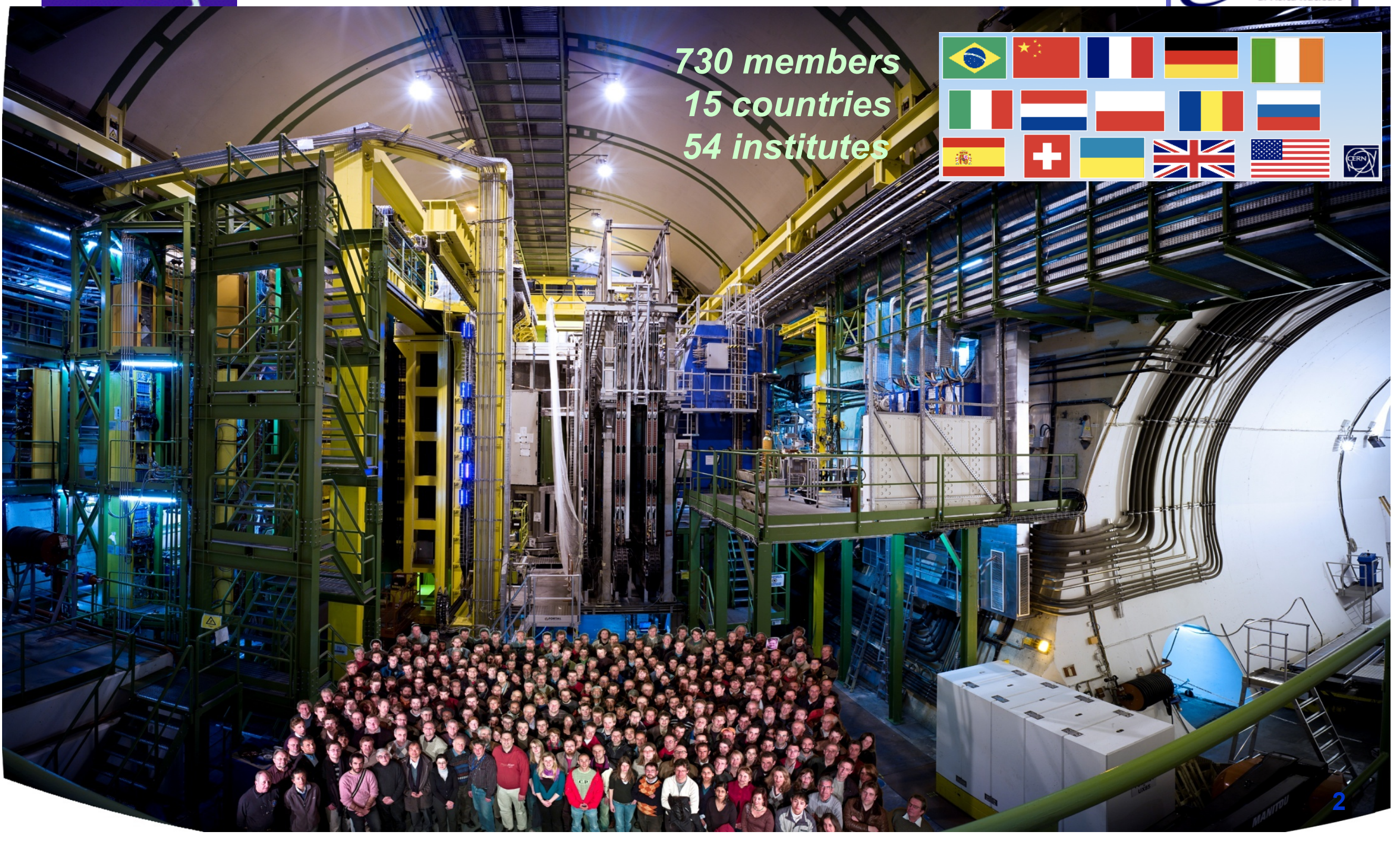




\section{IHCb Are we ready for B Physics? INFN}

- LHCb started physics run at $7 \mathrm{TeV}$ since March 31st: how far did we go for B Physics?

- Not so far yet, limited integrated luminosity, but

- Bulk of lumi delivered in a handful of solar days

- High efficiency of LHCb DAQ

- Detector and trigger fully operational

- First B candidates showing up

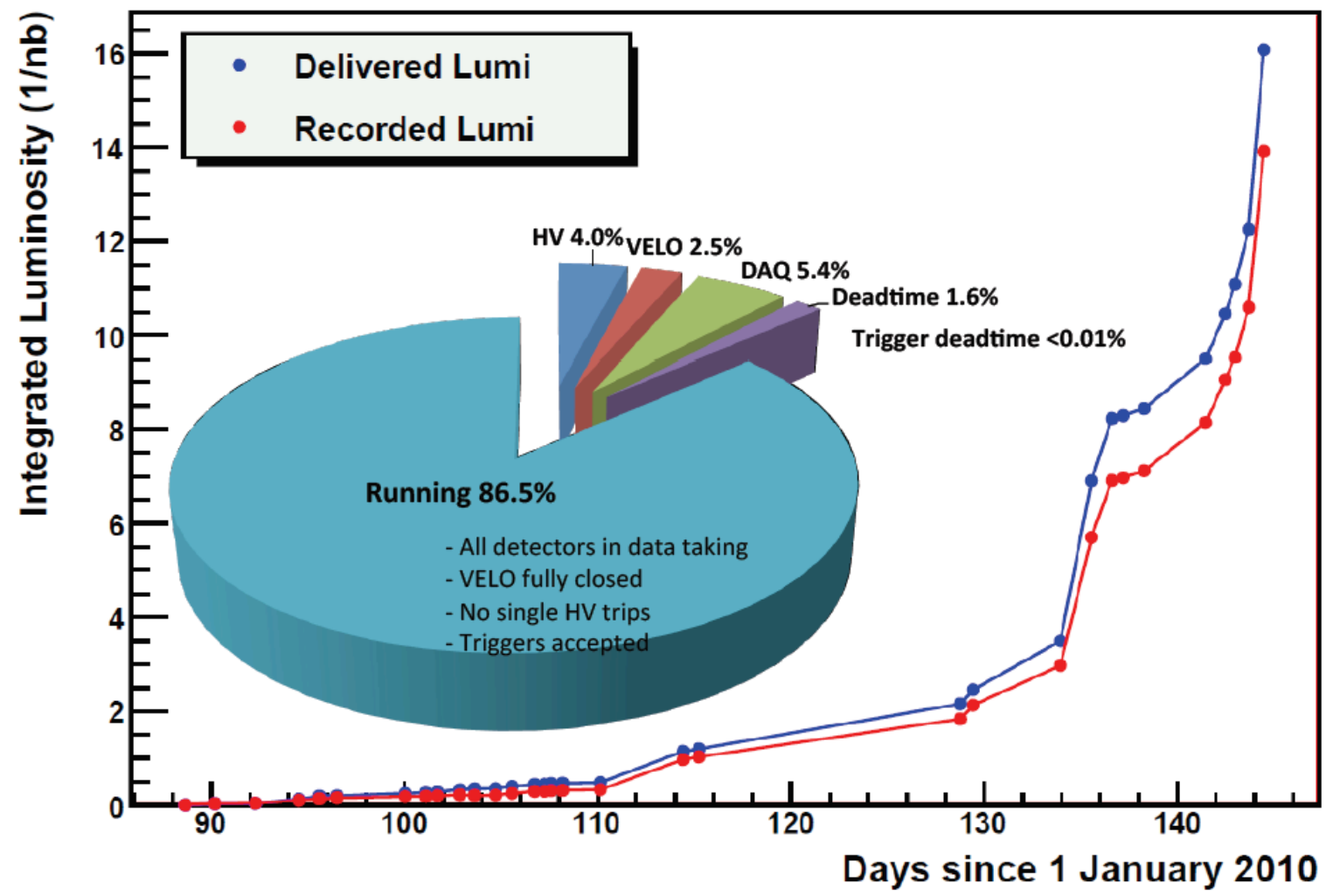

- It is just matter of how fast the machine will ramp up! 


\section{KHCD $\sqrt{s}=7 \mathrm{TeV}$ only? Don't panic INFN}

- Bottom and charm production cross sections just get a reduction of a factor $0.5 \rightarrow$ limited effect on physics reach

- What really matters, instead, is the real value of the beauty production cross section $\rightarrow$ measure it!
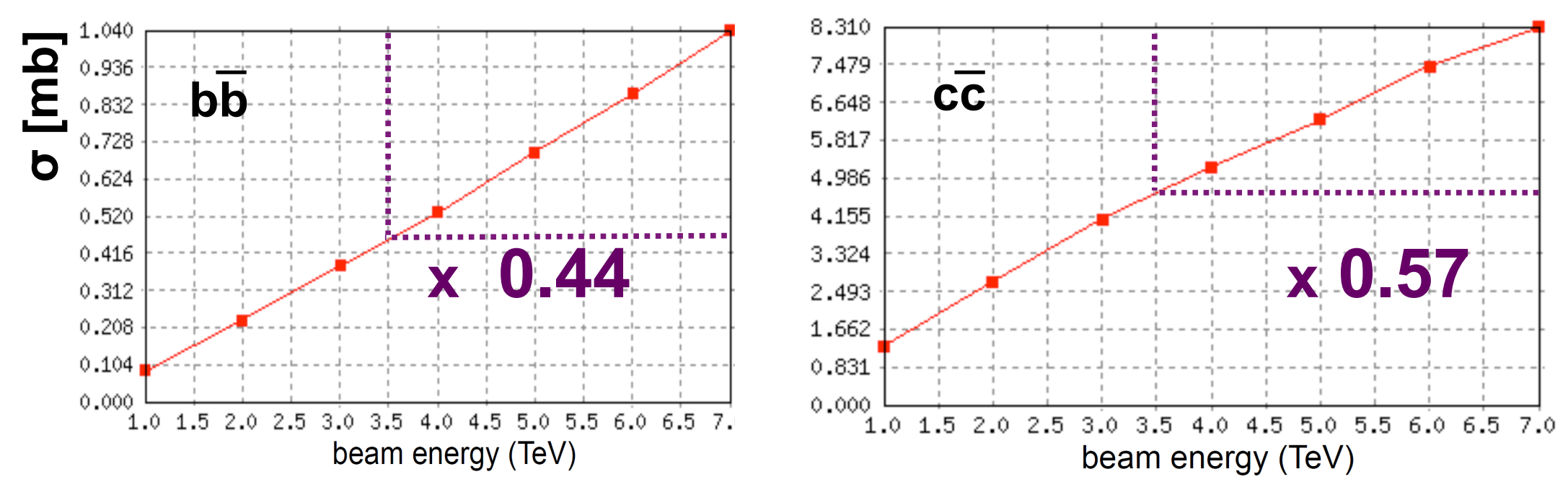

Scaling with energy predicted by Pythia 6.4 
- LHCb is a dedicated B physics experiment, though can do charming things as well

- Large beauty production cross section

- Expected in the range 200-500 $\mu$ b for a c.m.s. energy of 7-14 TeV

- All b-hadron zoology available

- $\mathrm{B}^{+}, \mathrm{B}^{0}, \mathrm{~B}_{\mathrm{s}}, \mathrm{B}_{\mathrm{c}}$, b-baryons

- LHCb acceptance optimised for forward $b \bar{b}$ production $\rightarrow$ Forward single arm spectrometer $1.9<\eta<4.9$

- b-hadrons produced at low angle

- Correlated $b \bar{b}$-production in same hemisphere 


\section{LHCh LHCb luminosity outlook}

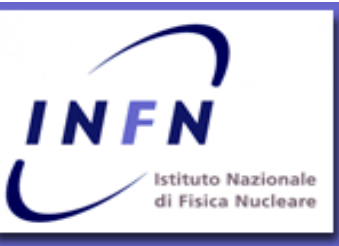

- Instantaneous lumi at IP8 intentionally moderated to $2 \times 10^{32}$ $\mathrm{cm}^{-1} \mathrm{~s}^{-2}$ (design value) in order to limit the number of multiple interactions per bunch crossing

- $2 \times 10^{32}$ is not a magic number $\rightarrow$ optimal running conditions could be even reached with somewhat higher lumi

- Nominal LHCb year $\rightarrow \mathrm{L}=2 \mathrm{fb}^{-1}$

- $10^{12} \mathrm{~b} \overline{\mathrm{b}}$ pairs at $14 \mathrm{TeV}$ in $4 \pi$

- In 2010 a first phase with a reduced number of bunches

- LHC could not exceed a lumi around $10^{31} \mathrm{~cm}^{-2} \mathrm{~s}^{-1}$

- Luminosity now rapidly increasing, although with still few bunches

- Many interactions per crossing $\rightarrow$ not ideal conditions, but still manageable

- Don't have a crystall ball, but the programme is to collect $200 \mathrm{pb}^{-1}$ in 2010 and $1 \mathrm{fb}^{-1}$ in 2011 


\section{LHCb LHCb Detector}

Tracking Station: $\mathrm{p}$ for lower energy tracks and long lived $V^{\circ}$ reconstruction

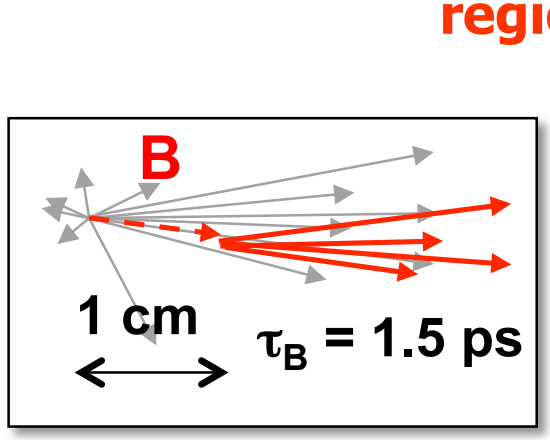

Interaction $5 \mathrm{~m}$ region

\section{VELO:}

primary vertex impact parameter displaced vertex

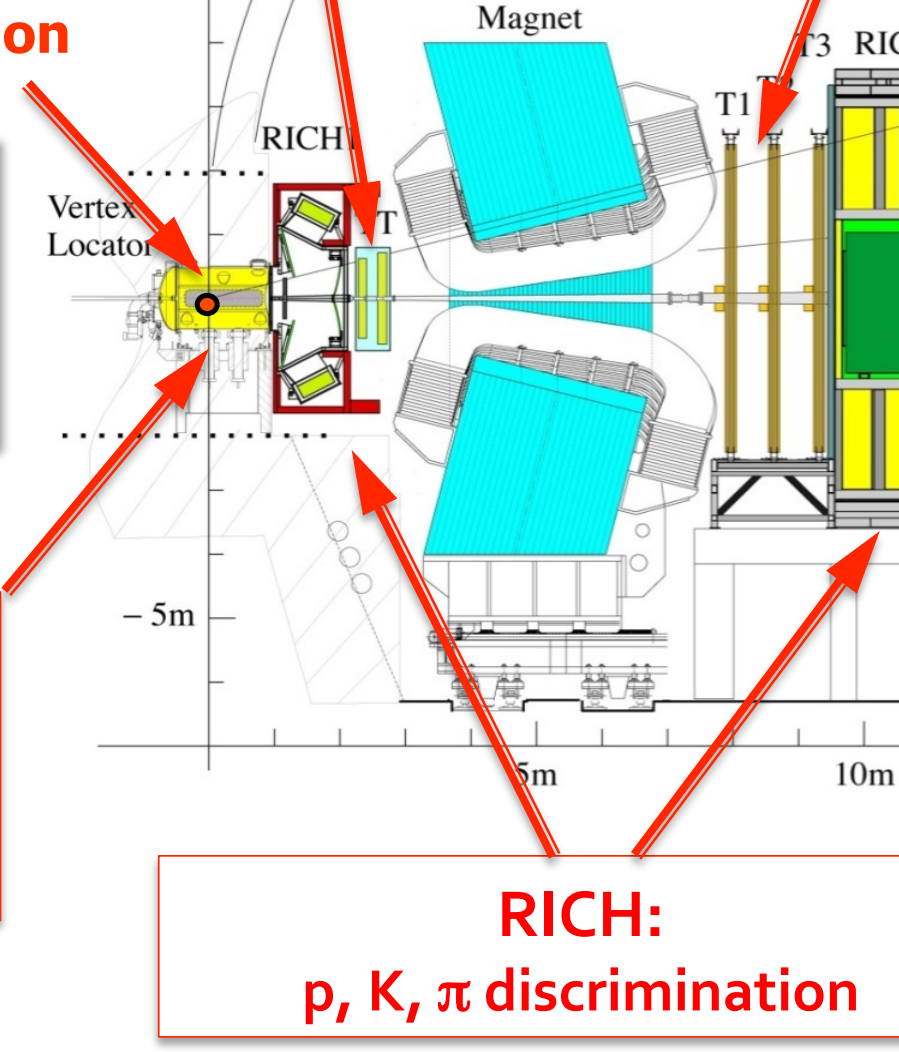

Tracking Stations: $p$ of charged particles that traverse the dipole magnet

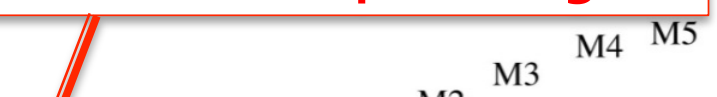

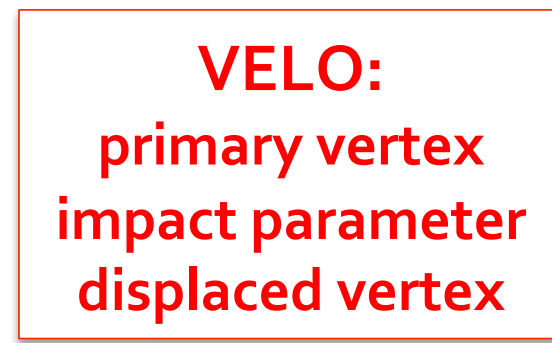

$p, K, \pi$ discrimination 


\section{KHCb \\ Vertexing is crucial for trigger and offline selection}

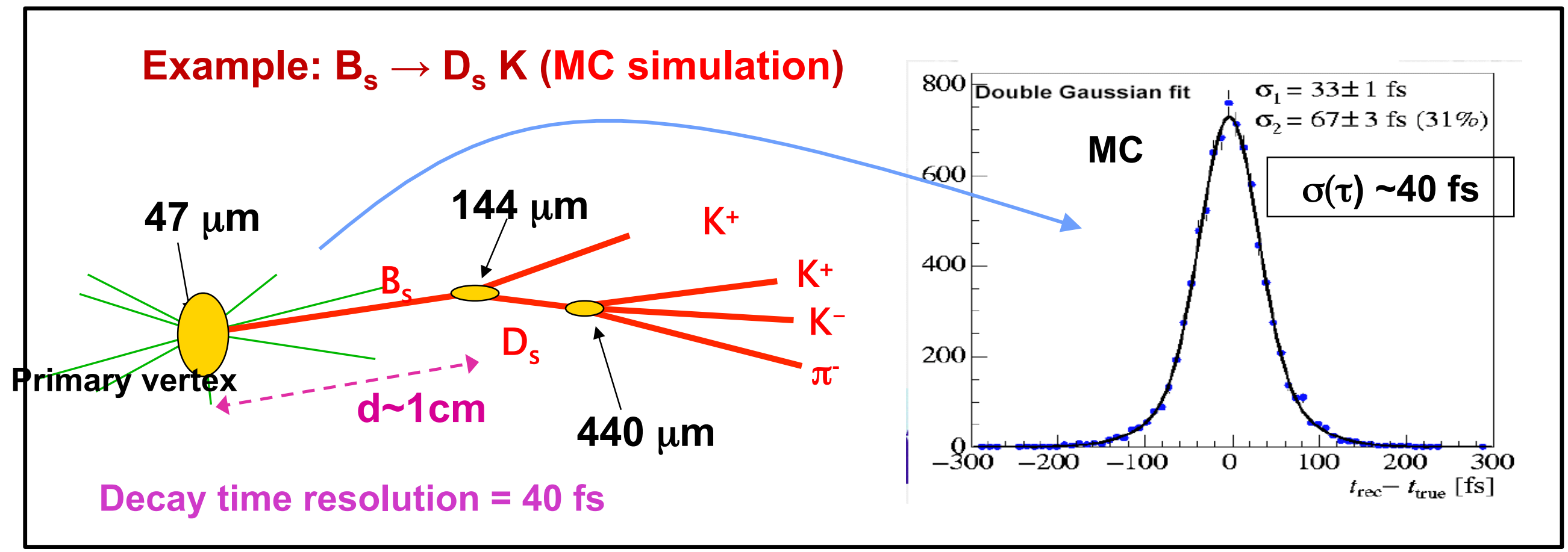

Trigger on impact parameter

Measurement of decay distance (and then proper decay time) 
- Primary Vertex (PV) is determined by fitting to a common vertex all the track segments reconstructed in the vertex detector

- Still about $40 \%$ difference between data and MC, but rapidly improving
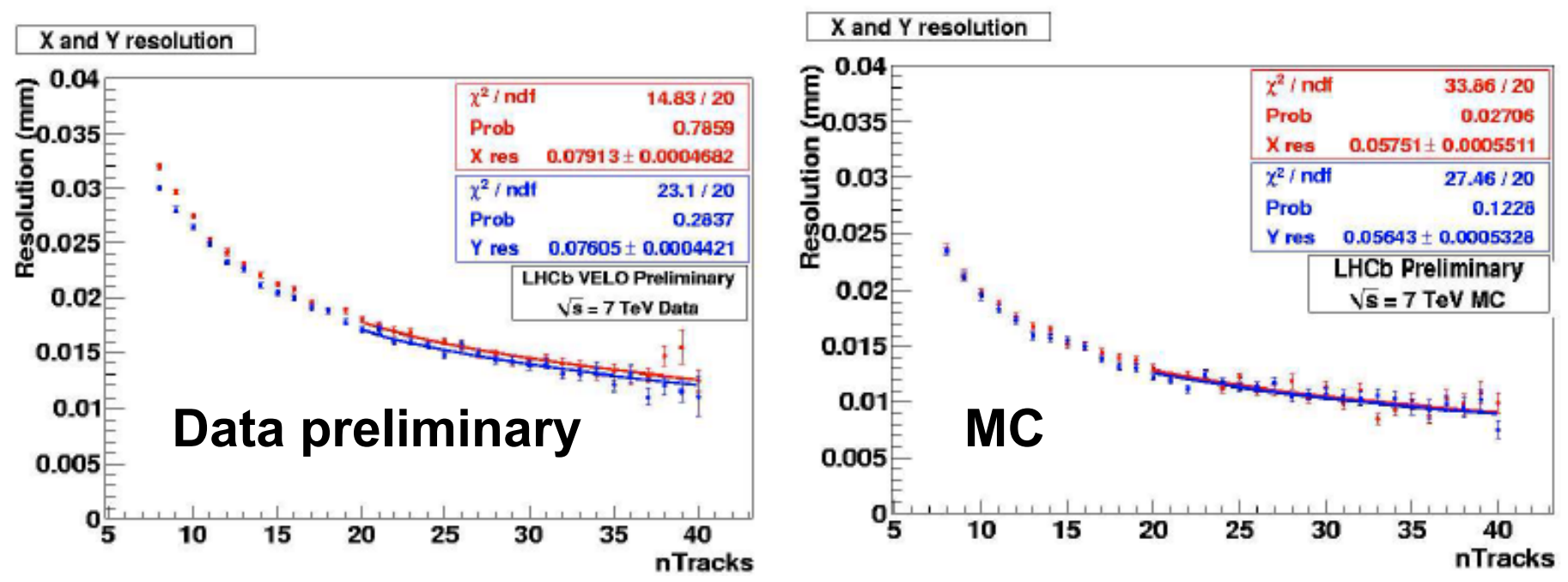

\begin{tabular}{|c|c|c|}
\hline & MC & Data \\
\hline$\Delta x(\mu m)$ & 11.5 & 15.8 \\
$\Delta y(\mu m)$ & 11.3 & 15.2 \\
$\Delta z(\mu m)$ & 57 & 91 \\
\hline
\end{tabular}

Average resolutions for $n$. tracks $=25$

$X$ and $Y$ resolutions as a function of the number of tracks forming the vertex 
- Impact parameter (IP)

- Closest approach of a track to a primary vertex

- IP resolution is mainly due to

- Multiple scattering in the detector material and beam pipe

- VELO misalignments and hit resolutions.

- 15-40\% discrepancy between MC and data

- Not dramatic, but lot of work going on to understand and fix some residual effects

Impact Parameter resolution in $\mathrm{Y}$

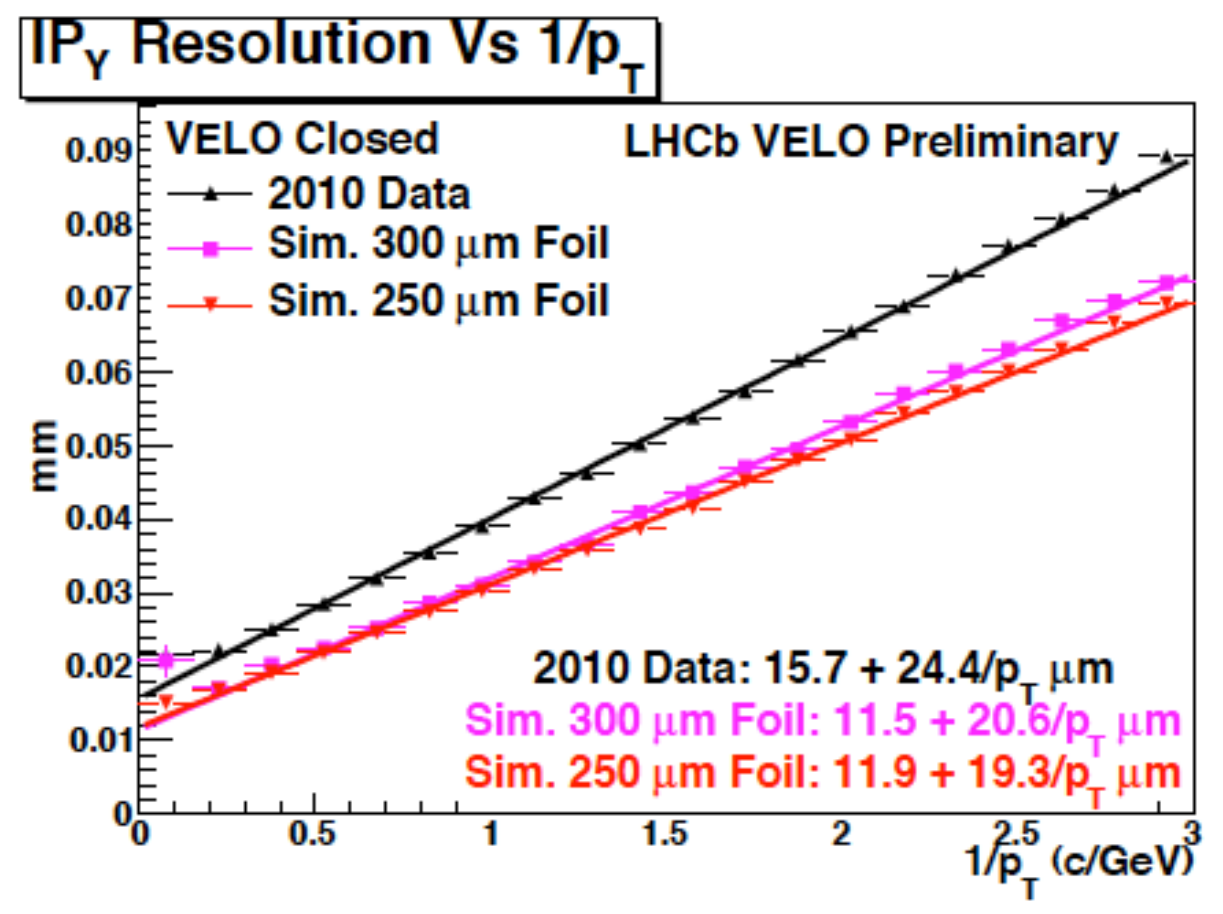



and $\mathrm{MC}$

- Good agreement between data and MC for track

Tracking efficiency vs $p_{T}$ inclusive distributions and efficiency

- Overall performances close to expectations
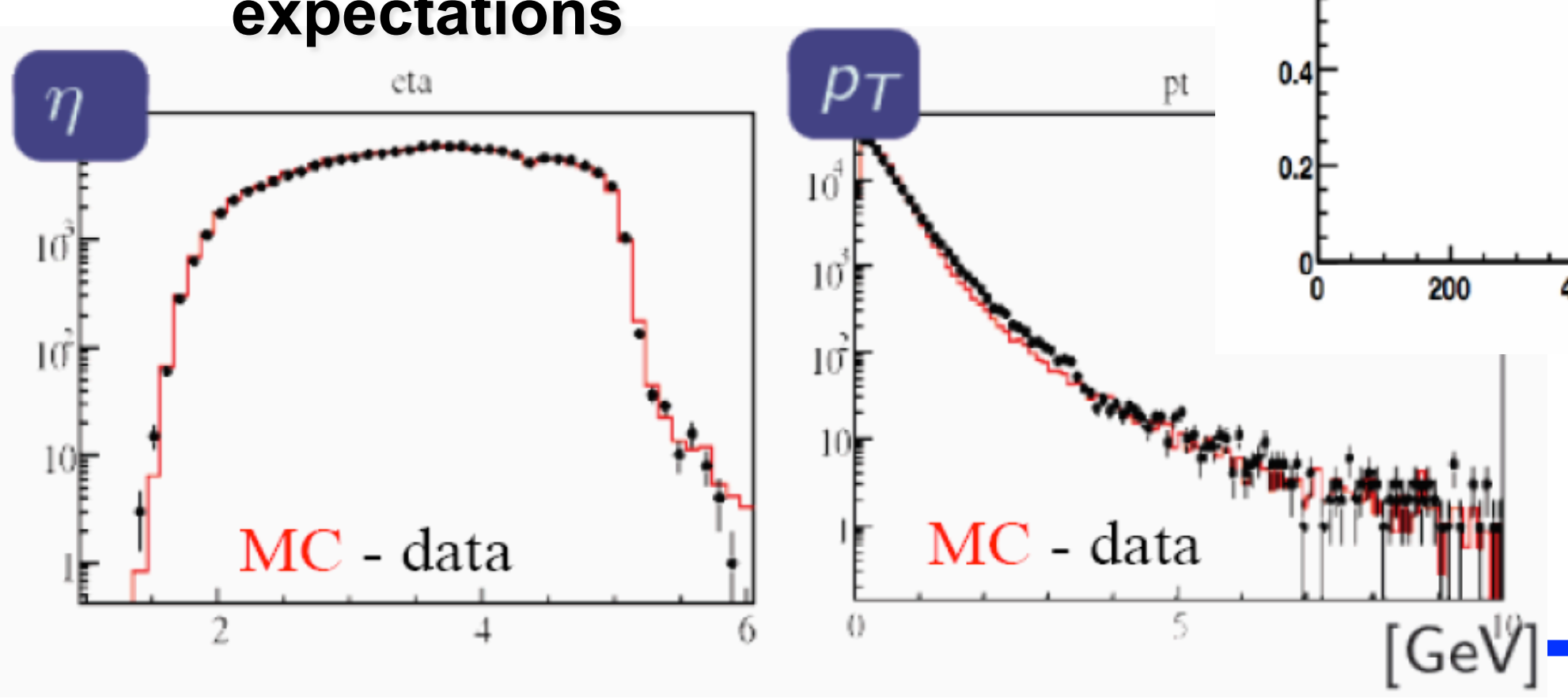

- Data

- Monte Carlo

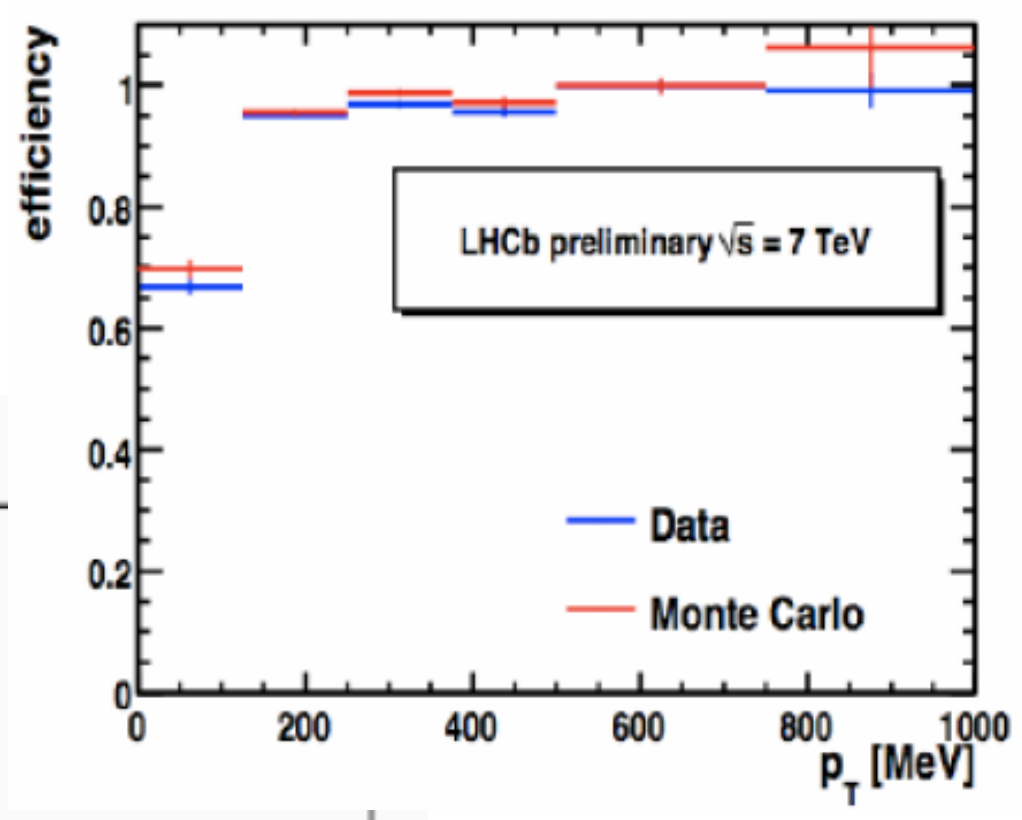

$\left[\mathrm{GeV}^{\mathrm{V}}\right]$ 


\section{Hadronic Particle Identification}

- RICH aligned with tracking system

- $\mathrm{K}$ and $\pi$ rings clearly visible

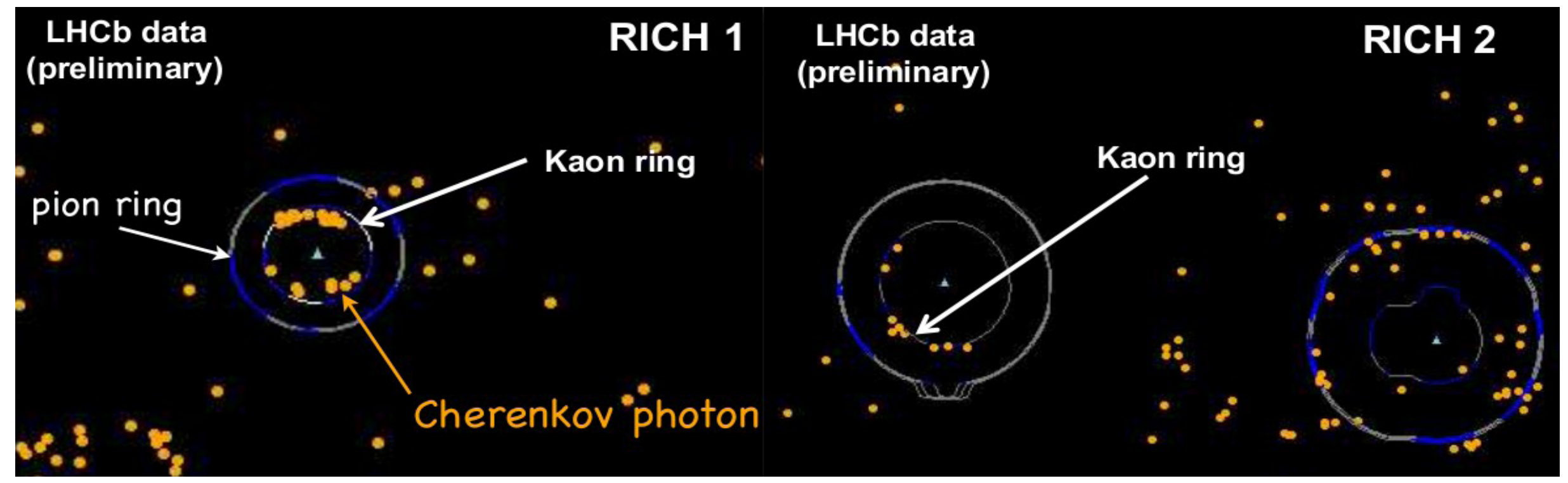

Calorimeter and Muon ID in Ulrik's talk 
Alignment and calibration work ongoing

- Angular resolutions close to expectations

- Efficiencies and misidentification rates will be calibrated with real data $\left(K_{s}, \Lambda, \phi\right.$ and $\left.D^{*}\right)$

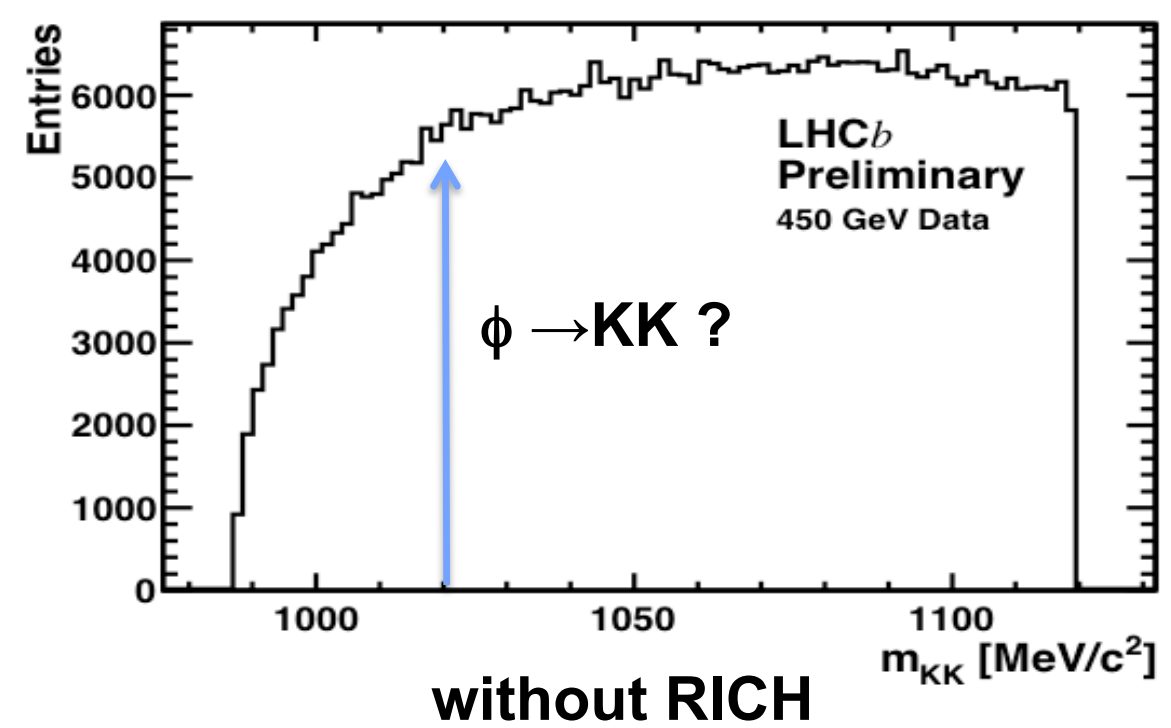

Efficiency and misid rate vs momentum for charged Kaons
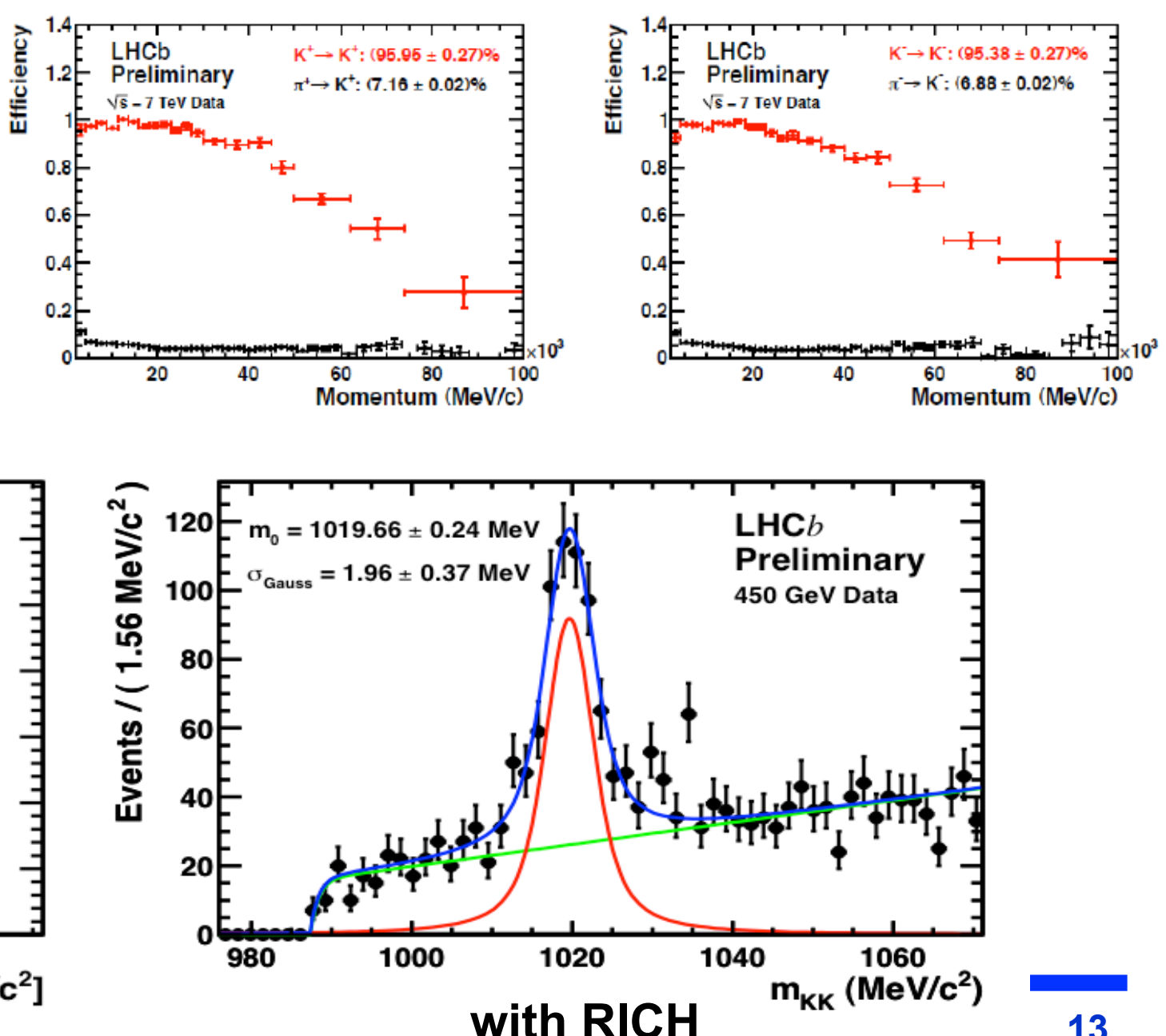


\section{LHCb THCP}

Significant samples of long lived strange particles starting to be available

- Long life allows selection of pure samples without need of PID observables $\rightarrow$ samples are to a large extent PID unbiased and can be used for calibrating RICH system response
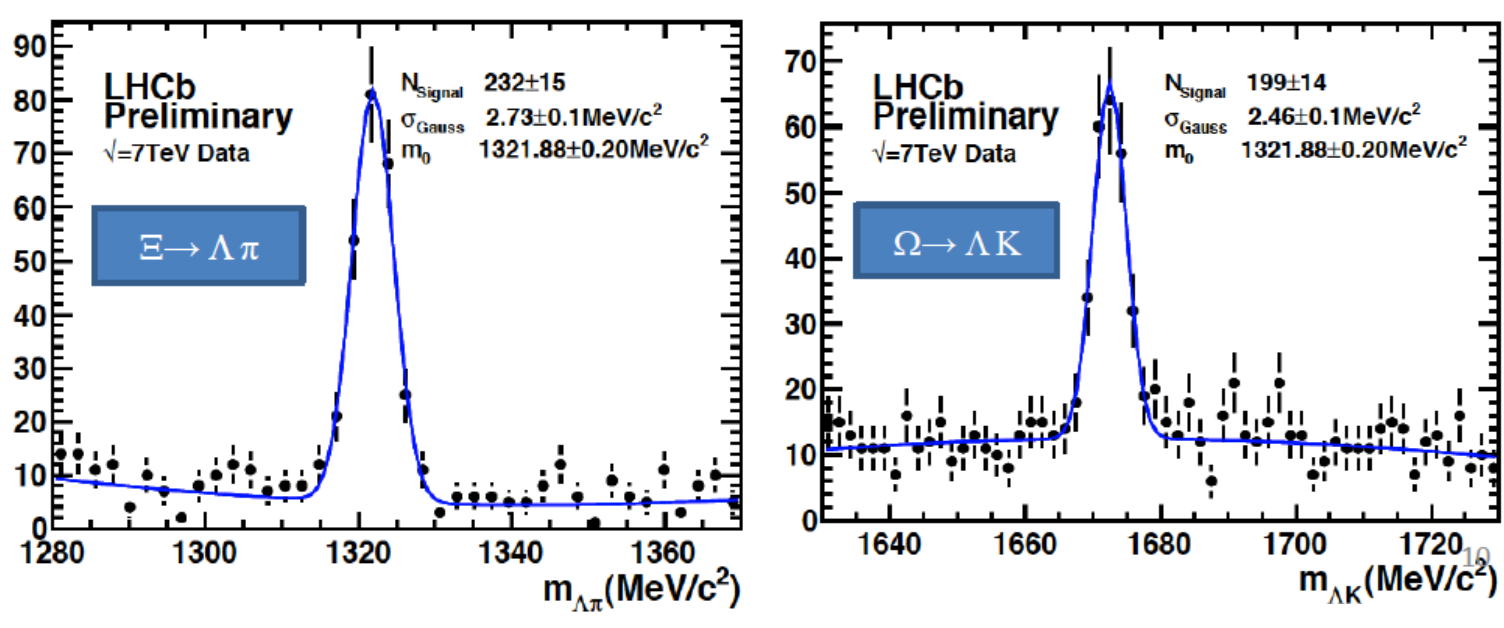
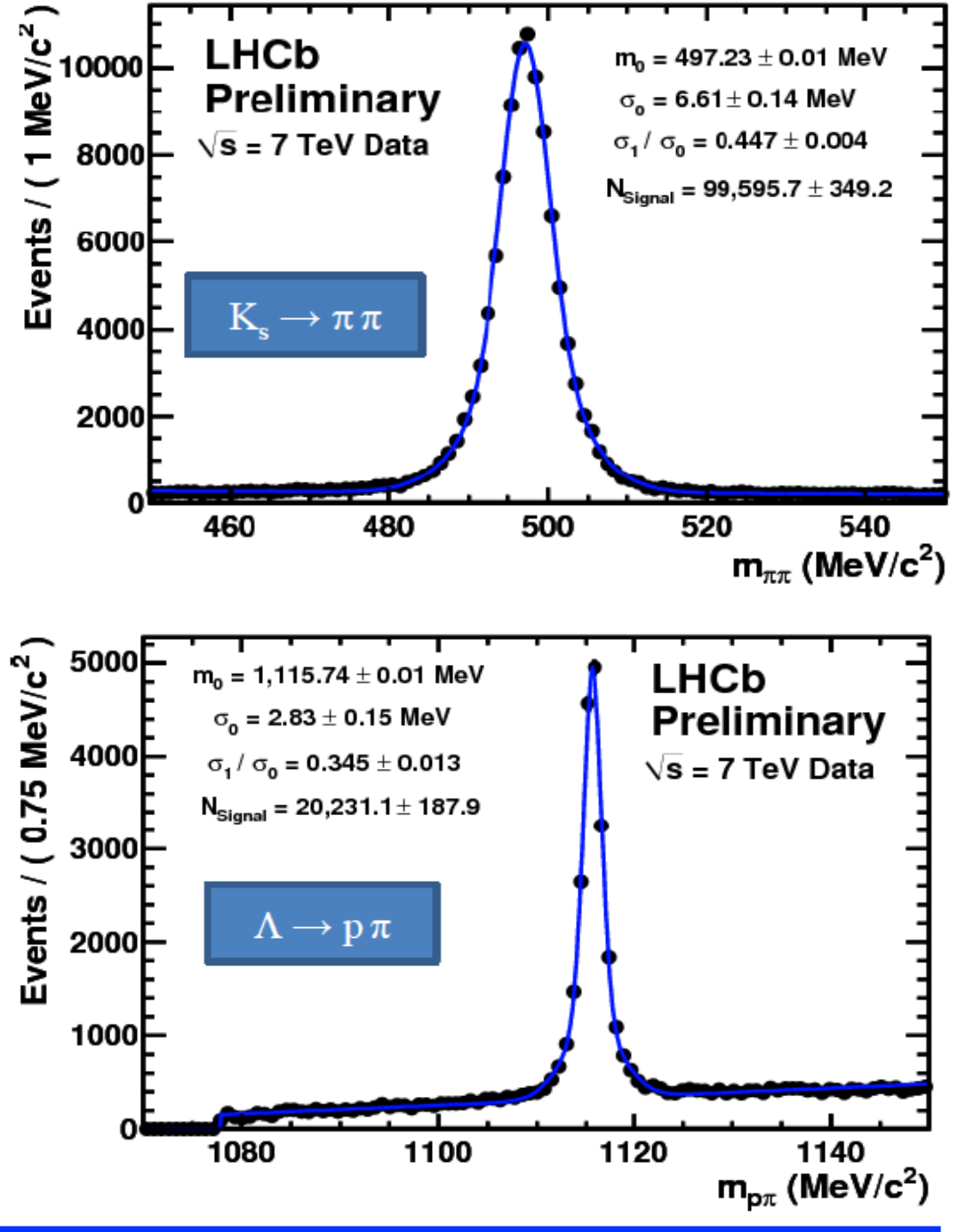


\section{LHCb \\ LHCb trigger design}

I N F N

- Using design

luminosity the trigger is optimized for $B$ physics

- But, low luminosity in 2010...

- some $10^{31} \mathrm{~cm}^{-2} \mathrm{~s}^{-1}$

- trigger thresholds can be relaxed $\rightarrow$ large gain in D efficiency $\rightarrow$ good year for charm physics!

charm had. B Lep. B

\begin{tabular}{l|l|l|l} 
Nominal & $10 \%$ & $40 \%$ & $90 \%$
\end{tabular}

lumi

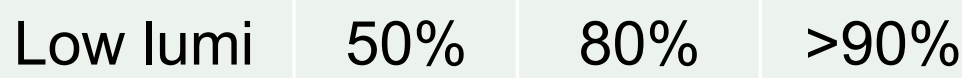

(2010)

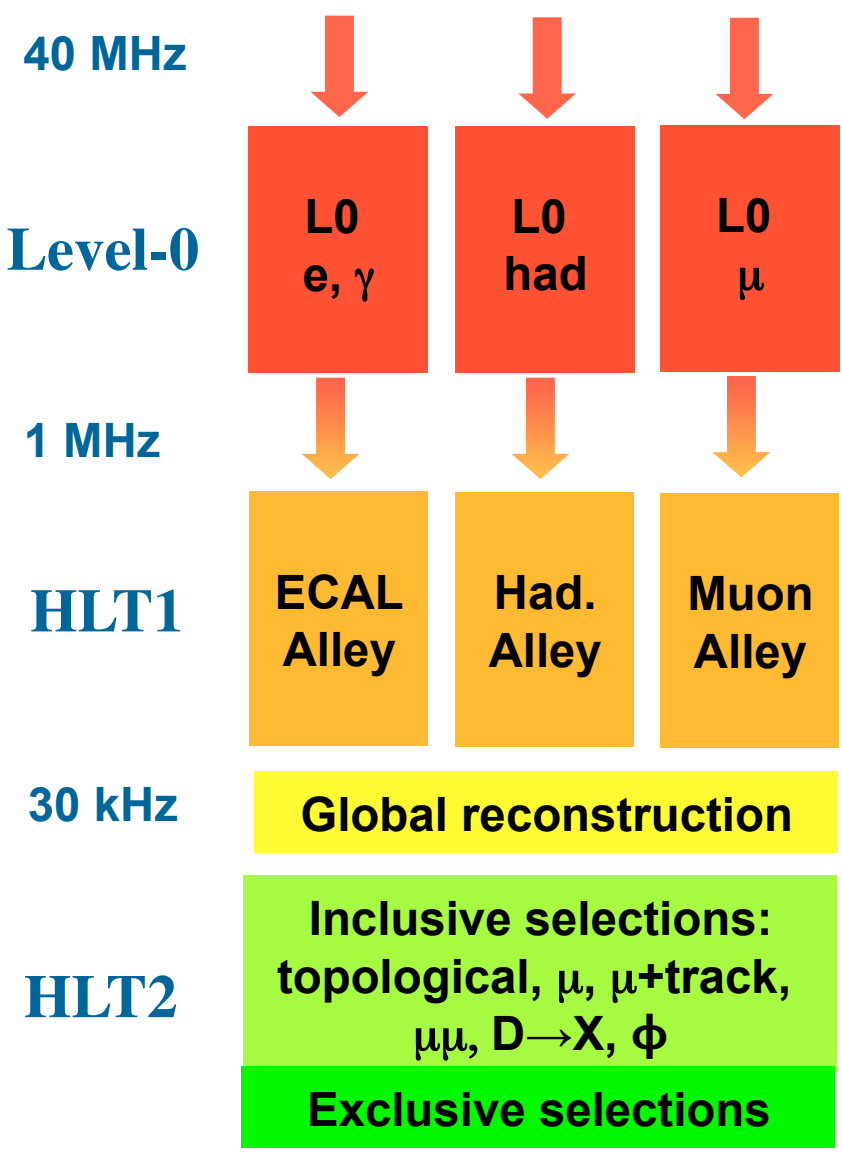

$2 \mathrm{kHz}$
Large transverse energy and momentum in calorimeter and muon systems

\section{Associate Level-0} signals with tracks, especially those in VELO displaced from Primary Vertex

Full detector info available for inclusive and exclusive selections 


\section{Trigger startup in this early phase}

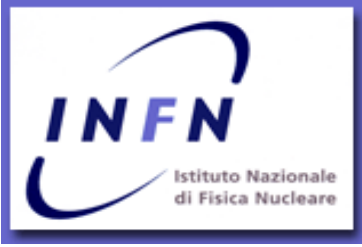

\begin{tabular}{|c|c|c|c|}
\hline Interaction rate & LO output rate & HLT1 output rate & HLT2 output rate \\
\hline Up to $2 \mathrm{kHz}$ & Up to $2 \mathrm{kHz}$ & & \\
\hline Up to $25 \mathrm{kHz}$ & Up to $25 \mathrm{kHz}$ & $2 \mathrm{kHz}$ & \\
\hline Up to $300 \mathrm{kHz}$ & Up to $300 \mathrm{kHz}$ & $10 \mathrm{kHz}$ & $2 \mathrm{kHz}$ \\
\hline
\end{tabular}

- L0 always passing through so far

- minimum bias and random triggers

- Couple of weeks of HLT1 exercising real rejection

- During summer expect 2 months of continuous running

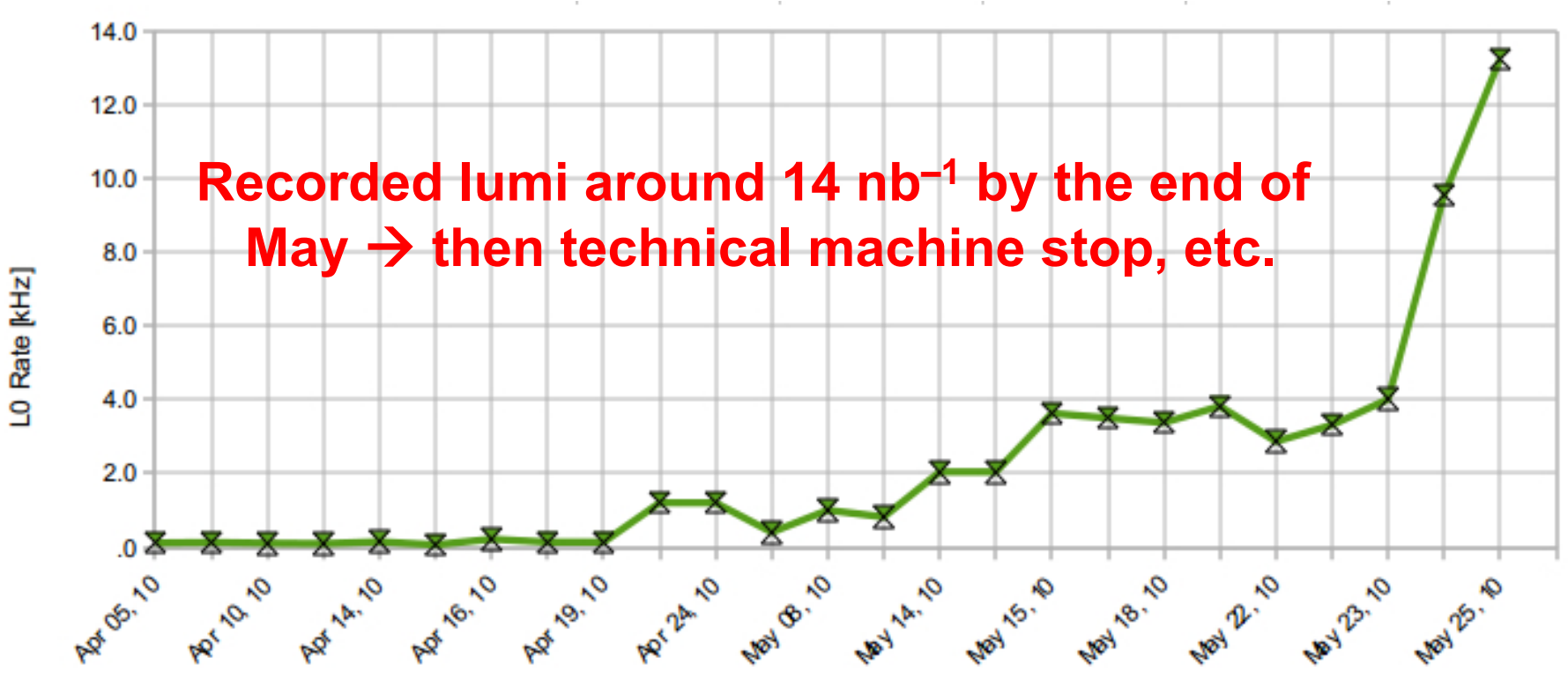

- Lot of stat. expected 


\section{LHCb B physics programme Main Items}

- Selected key measurements

- Search for $B_{s} \rightarrow \mu \mu$

- Mixing-induced CP violation in $B_{s} \rightarrow J / \Psi \varphi, B_{s} \rightarrow \varphi \varphi, \ldots$

- Charmless 2-body $B$ decays

- CKM angle $Y$ from tree-level $B$ decays

- $B_{s} \rightarrow \varphi Y$ and other radiative $B$ decays

- Asymmetries in $\mathrm{B}^{0} \rightarrow \mathrm{K}^{*} \mathrm{l}^{+}-$decays

- Roadmap note

- LHCb-PUB-2009-029, arXiv:0912.4179v2 [hep-ex], Feb 2010

- Nominal assumptions there:

- $2 \mathrm{fb}^{-1}$ per year at $25 \mathrm{~ns}$ bunch spacing

- $\sqrt{ } \mathrm{s}=14 \mathrm{TeV}$

- Beauty production cross section $500 \mu b$ 


\section{KHCb 2010-2011 physics roadmap}

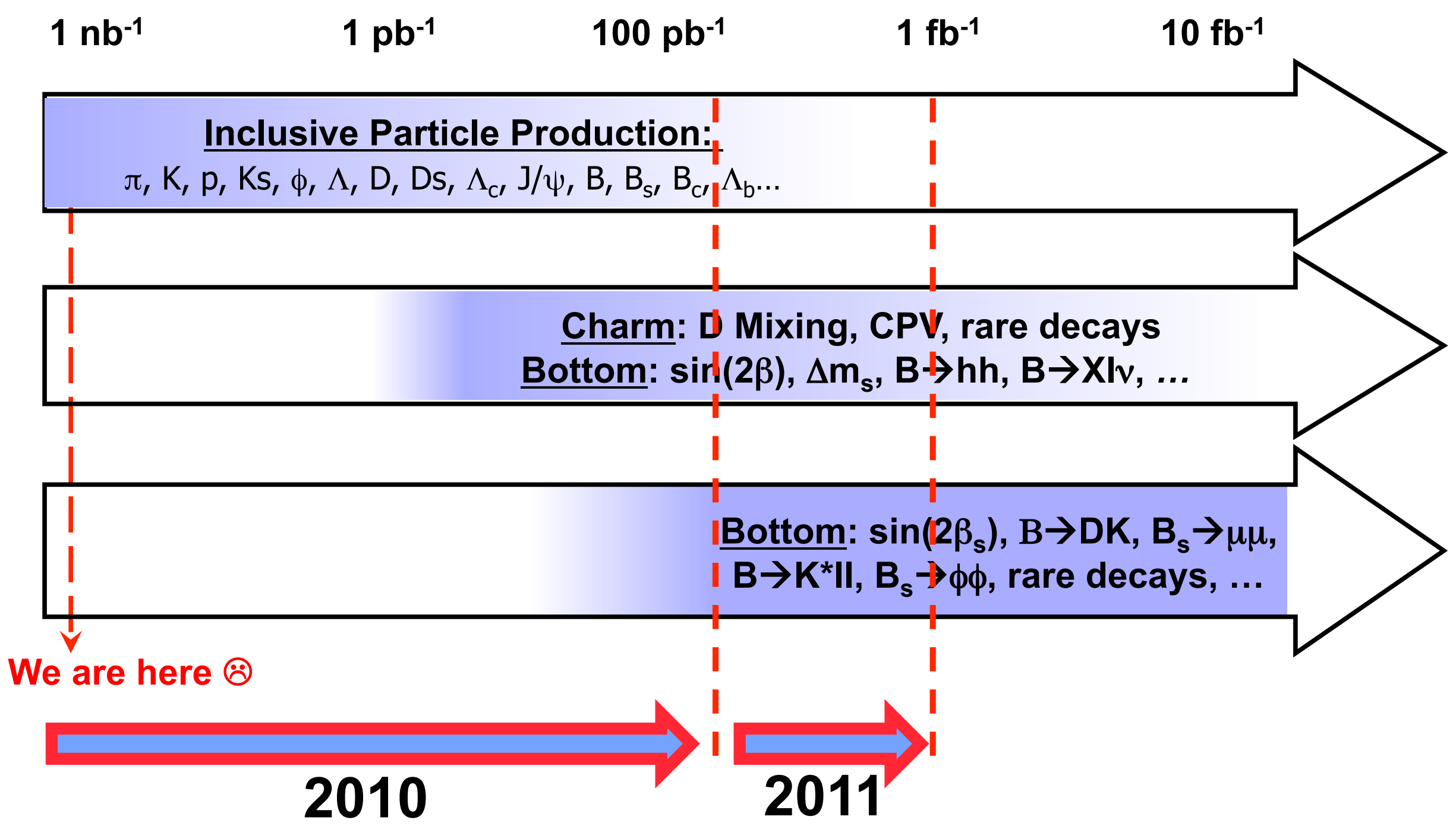




\section{LHCb Prompt $\mathrm{K}_{\mathrm{S}}$ production (2009)}
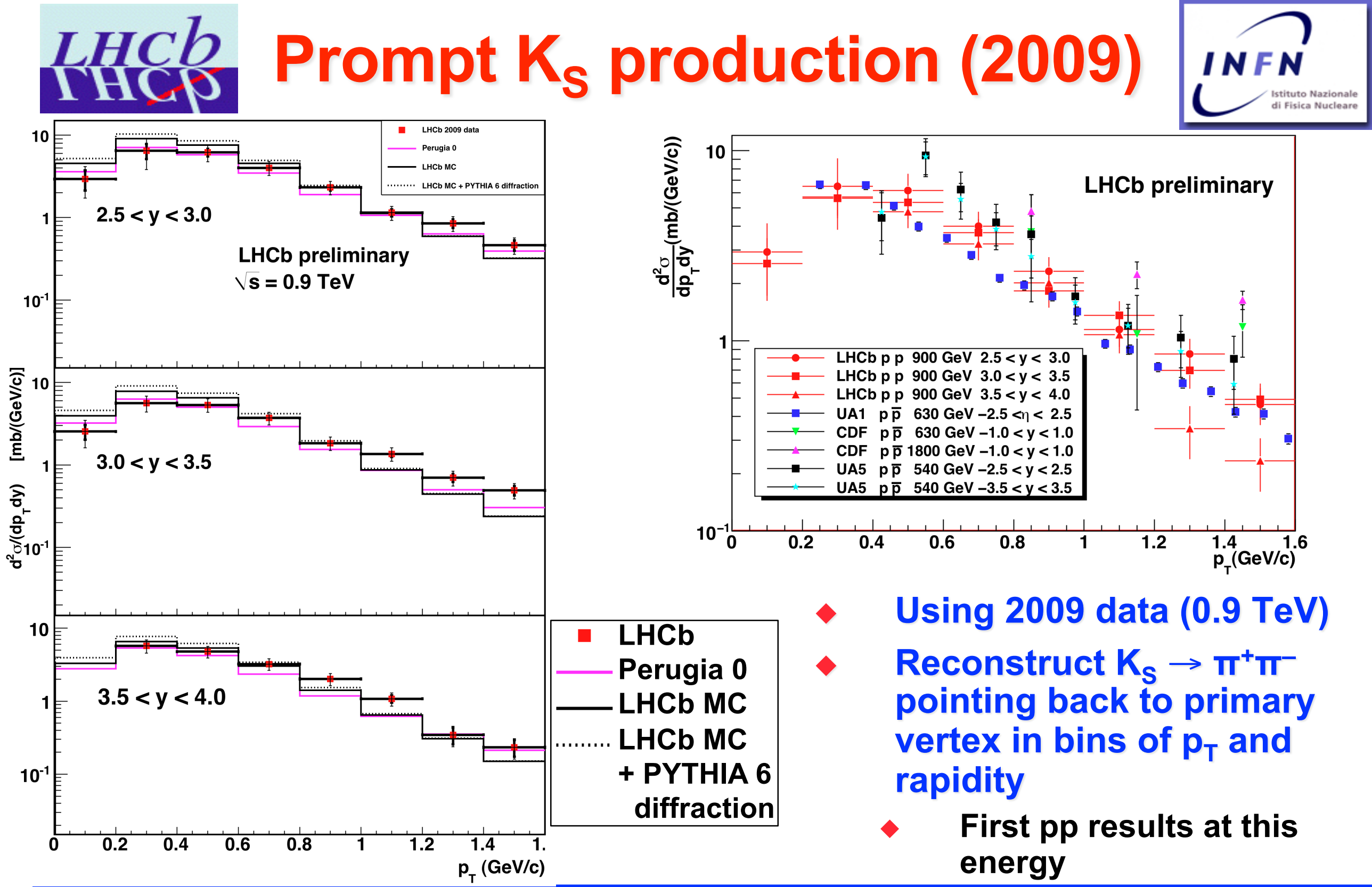

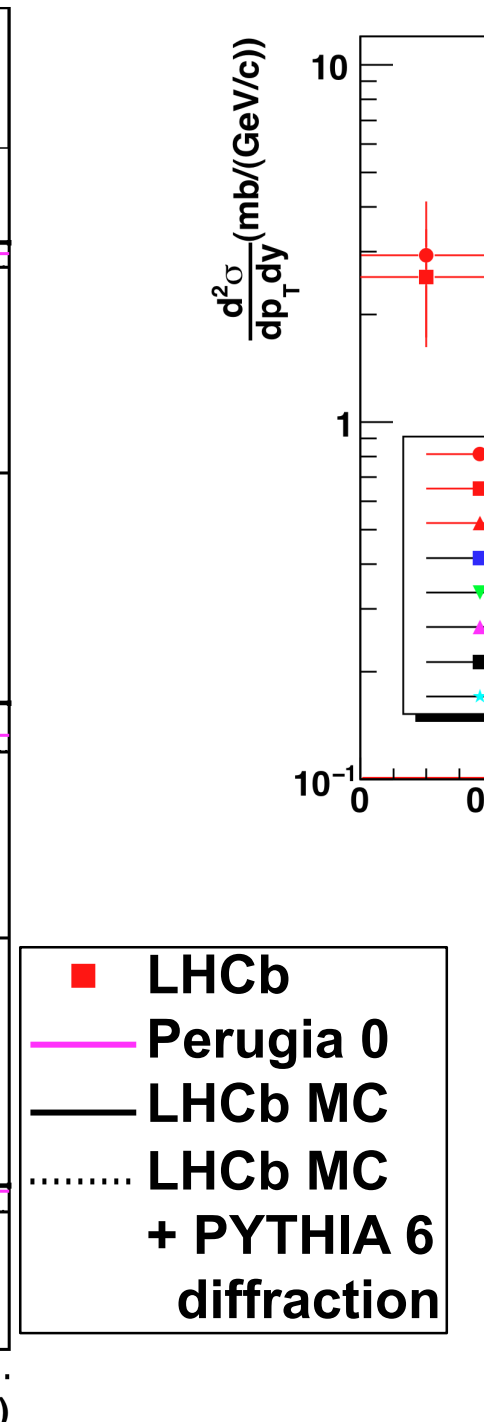

- Using 2009 data (0.9 TeV)

Reconstruct $\mathrm{K}_{\mathrm{S}} \rightarrow \pi^{+} \pi^{-}$ pointing back to primary vertex in bins of $p_{T}$ and rapidity

First pp results at this energy 


\section{LHCb \\ Prompt $\Lambda$ and $\bar{\Lambda}$ production}

I N F N

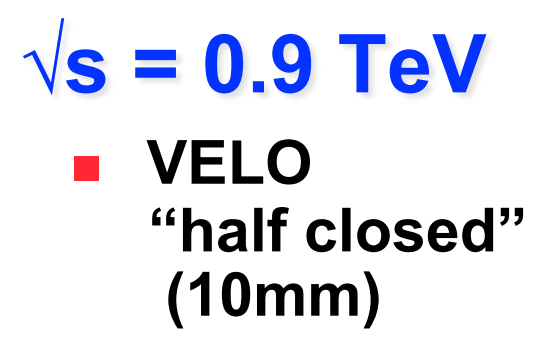

- VELO

"half closed" $(10 \mathrm{~mm})$

$$
\begin{aligned}
& \sqrt{s}=7 \mathrm{TeV} \\
& \text { - VELO closed }
\end{aligned}
$$
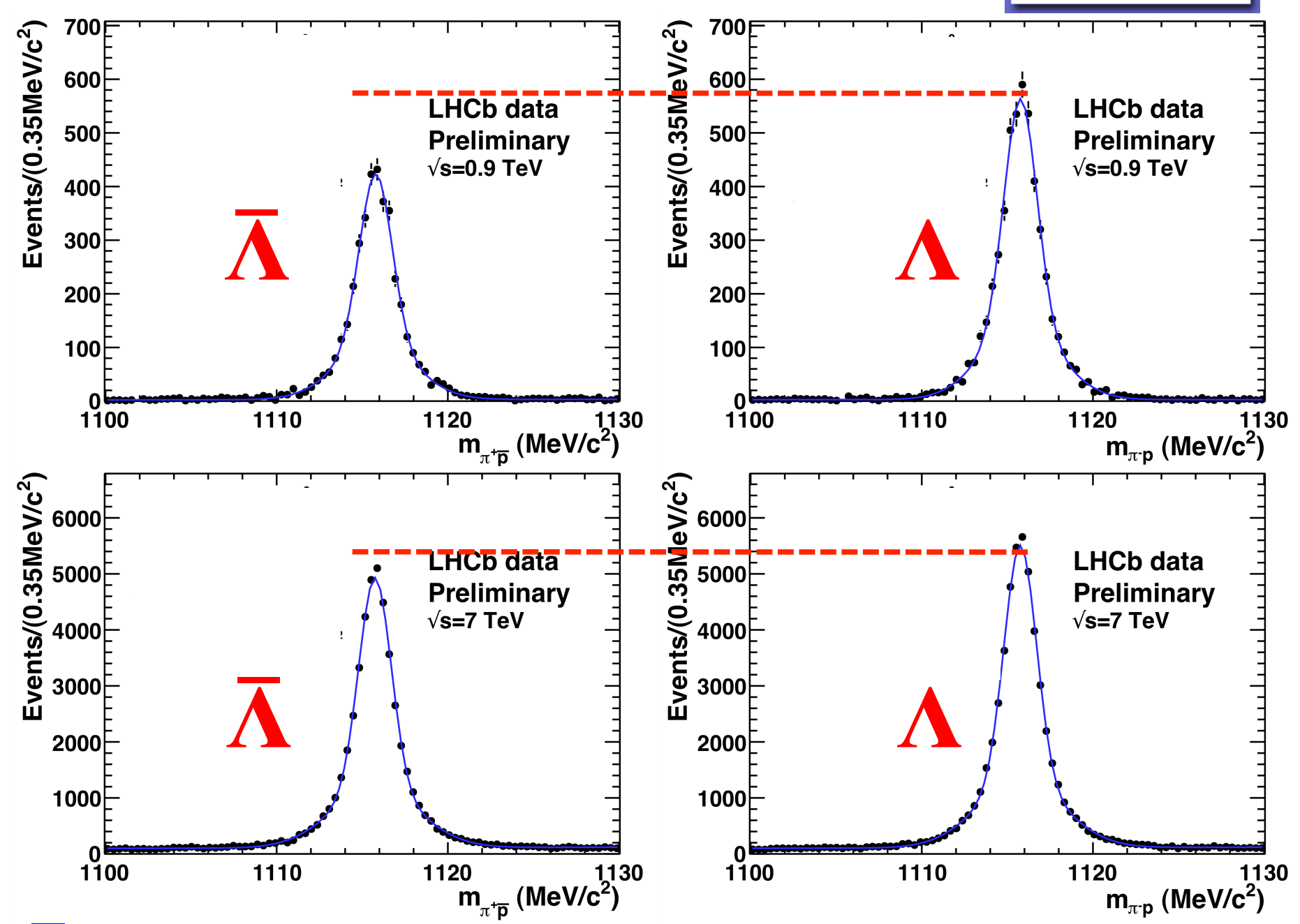

no PID used: $\Lambda \bar{\Lambda}$ separated on Armenteros-Podolansky plot 


\section{$\mathrm{LHCl}$ First clean heavy flavour THCP signals available (not yet B eh!)}

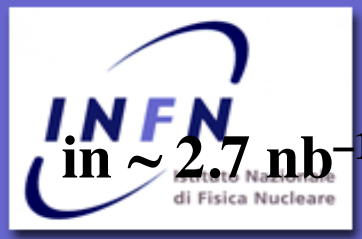

- Clean sample of $D^{0} \rightarrow h^{+} h^{-}$

- Simple check: measurement of $\mathrm{D}^{0}$ lifetime

- Use pure $D^{0} \rightarrow K^{-} \pi^{+}$selection $(S / B \sim 22)$

- Simple minded approach for the moment

- Fit only the tail of the proper time distribution, where efficiency is constant

- $\mathrm{T}\left(\mathrm{D}^{0}\right)=0.398 \pm 0.026 \mathrm{ps}$ (stat. only)

- Good agreement with PDG: $0.4101 \pm 0.0015$ ps
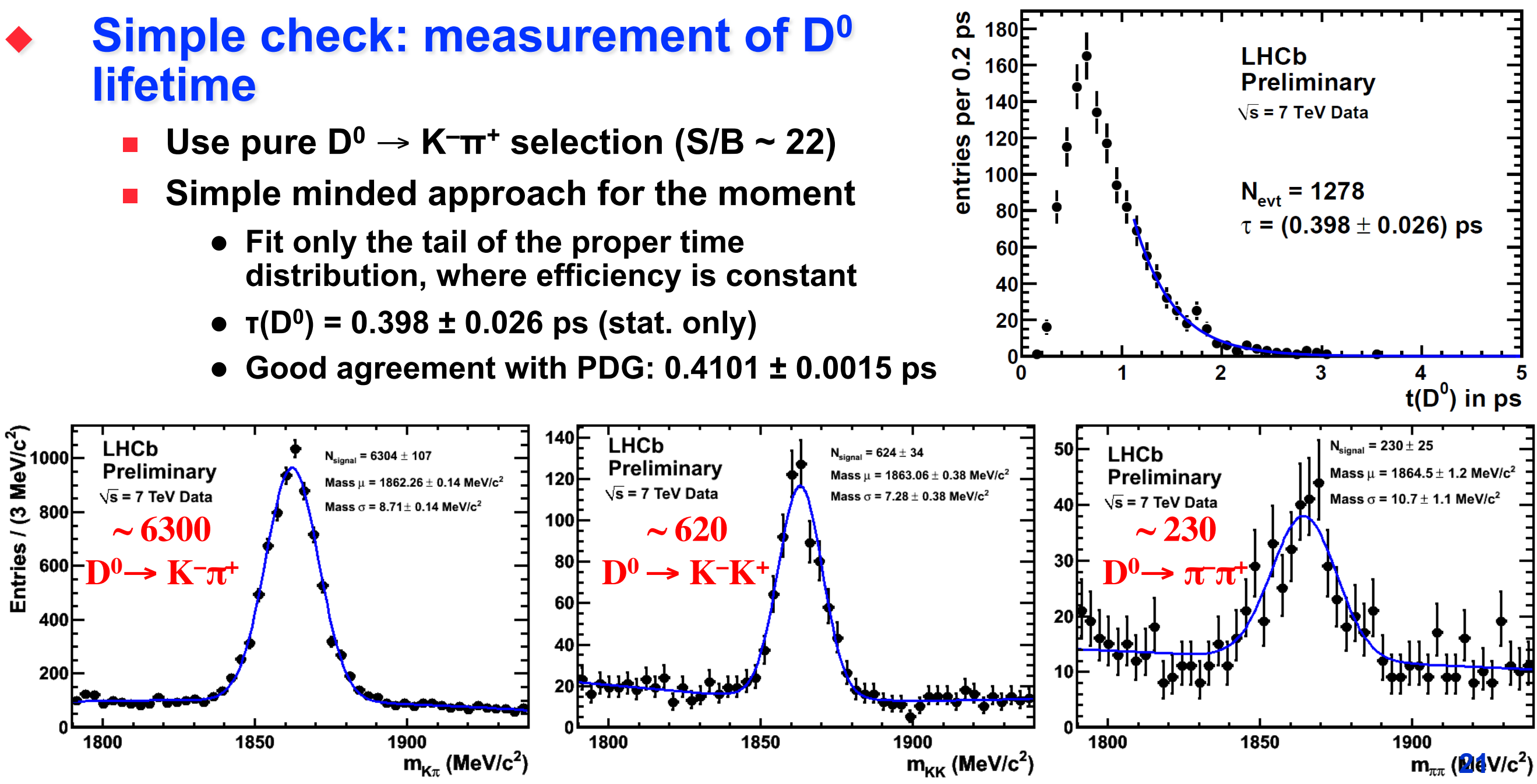


\section{$\mathrm{LHCb}$ Direct CP violation studies in the charm sector}

- Relevant modes

- Singly Cabibbo-suppressed decays, where NP may enter in gluonic Penguin

- 3-body decays with Dalitz plot analyses

$\rightarrow \mathrm{D}^{+} \rightarrow \mathrm{K}^{+} \mathrm{K}^{-} \mathrm{T}^{+}$is an excellent mode

- With also the good feature of having Cabibbo-favoured $D_{s}^{+} \rightarrow K^{+} K^{-} \pi^{+}$and $D^{+} \rightarrow K^{+} \pi^{-} \Pi^{+}$decays to be used as control channels
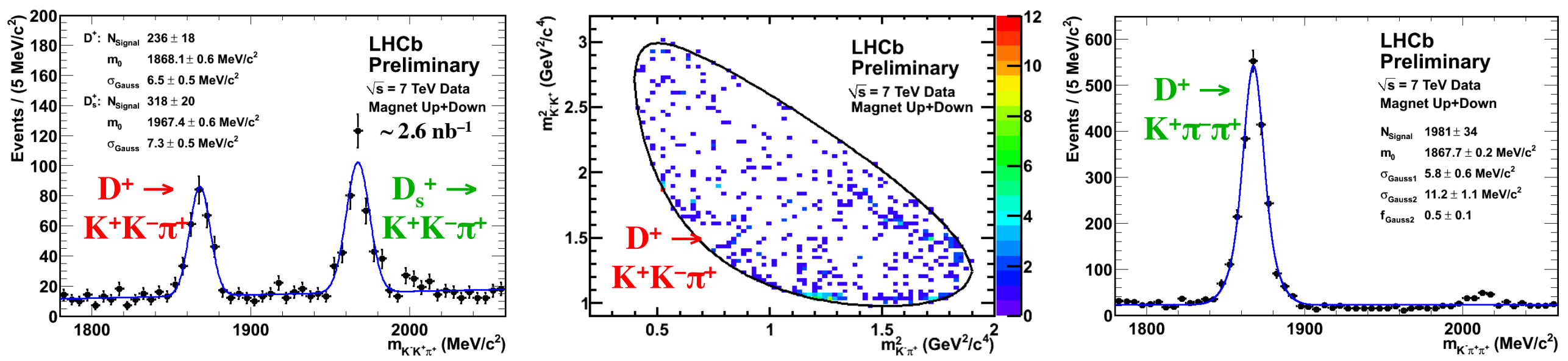

- About 5 million events ere expected in $0.1 \mathrm{fb}^{-1}$

- an order of magnitude more than B-factory samples 


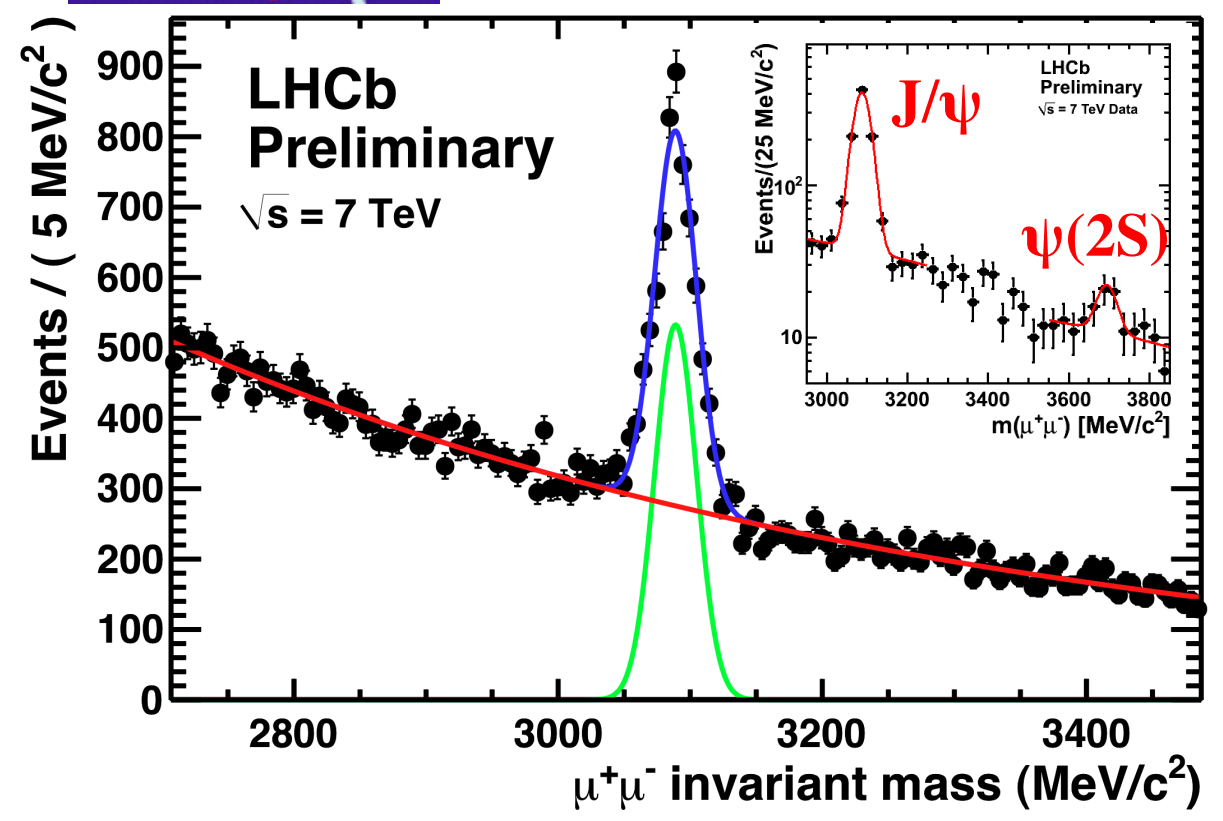

- $J / \Psi$ signal very rich $\rightarrow$ extremely important for calibration issues

- alignment, tracking studies, proper time calibration

- momentum resolution, mass scale

- Muon identification

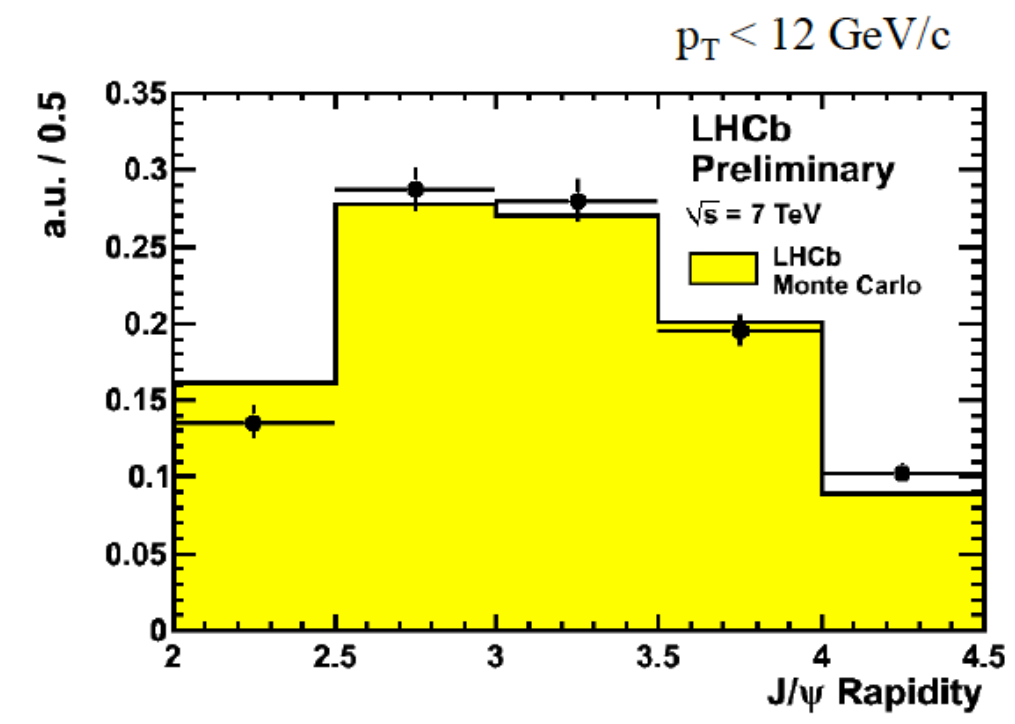

$\Psi$ 's play a central role in the LHCb

physics programme

- quarkonium production, polarization, etc.

- bottom physics with both inclusive and exclusive and $b \rightarrow J / \Psi$ decays 


\section{LHCP $\mathrm{J} / \Psi$ pseudo proper time}

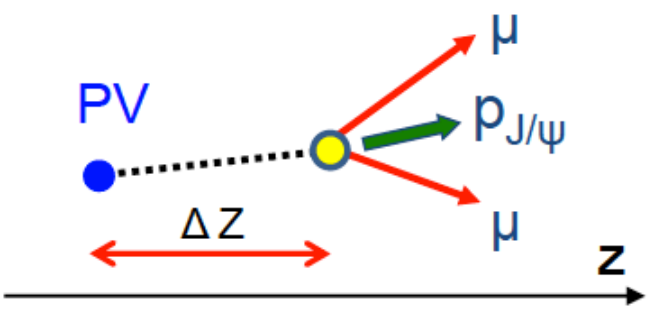

$t_{z}$ pesudo proper time

$$
\mathrm{t}_{\mathrm{z}}=\left(\mathrm{z}_{\mathrm{J} / \psi}-\mathrm{z}_{\mathrm{PV}}\right) \frac{\mathrm{m}_{\mathrm{J} / \psi}}{\mathrm{p}_{\mathrm{z}, \mathrm{J} / \psi}}
$$

- Long tail due to long-lived signals from decays of B hadrons
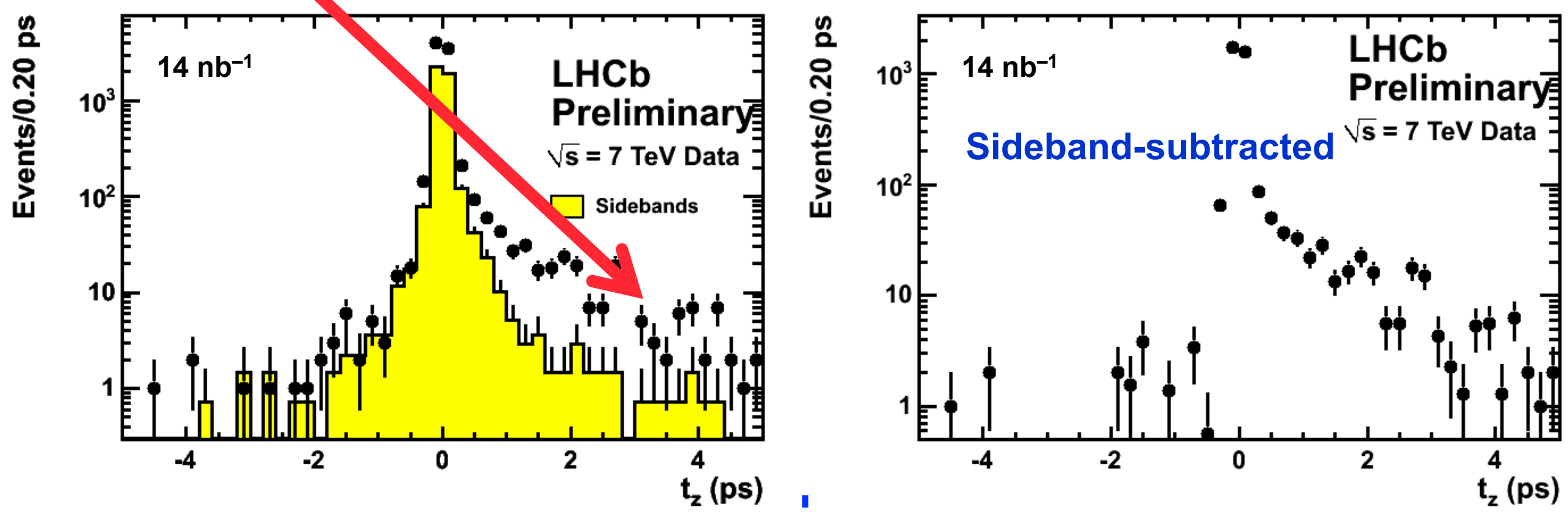


\section{IHCb \\ $\mathrm{B}^{+} \rightarrow \mathrm{J} / \boldsymbol{\Psi} \mathrm{K}^{+}$candidate ( $\mathrm{x}-\mathrm{y}$ plane projection)}
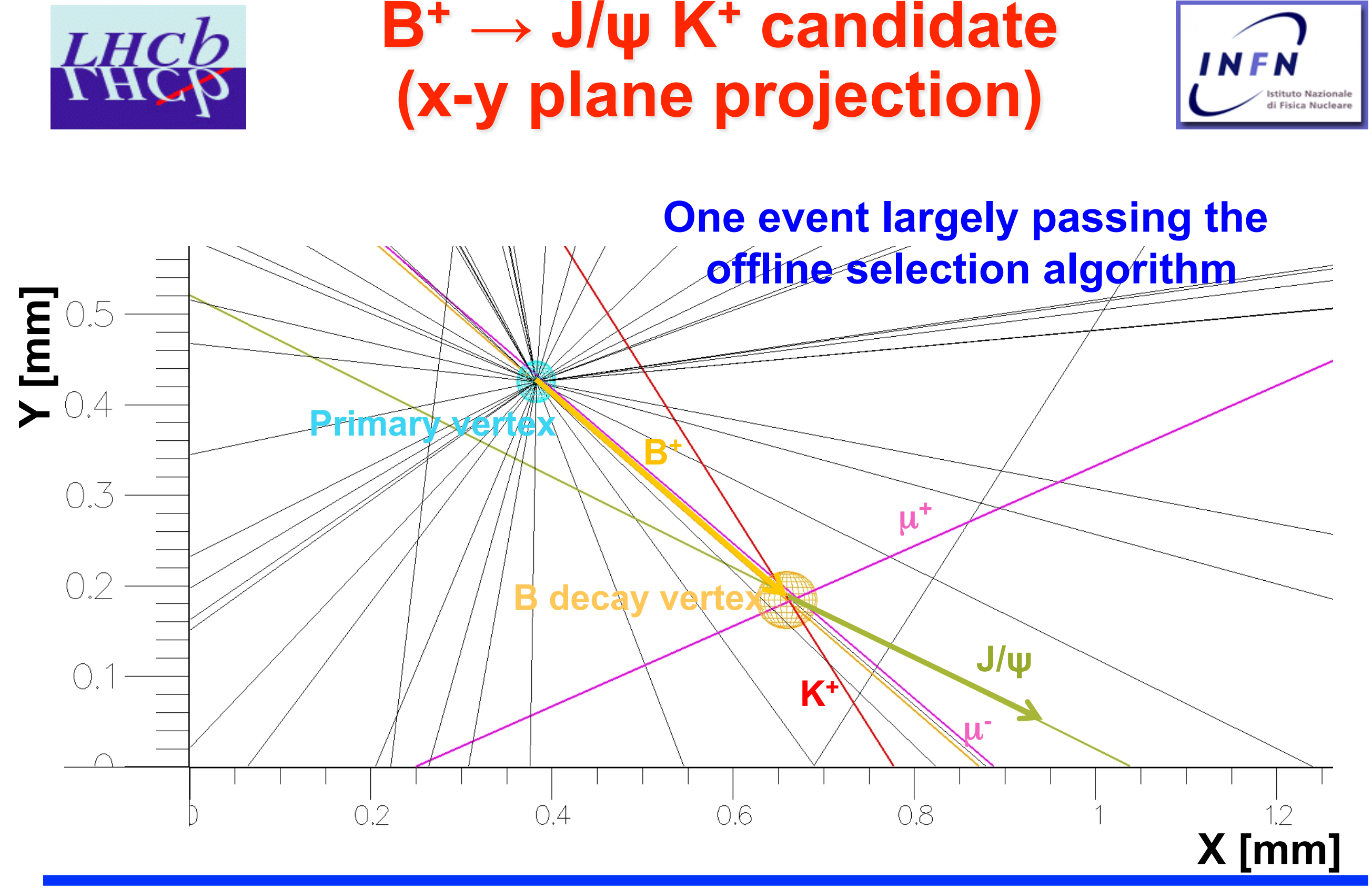


\section{IHCh Exclusive hadronic B decays}

First signal seen combining two modes $\mathrm{B}^{0} \rightarrow \mathrm{D}^{+} \pi^{-}$and $\mathrm{B}^{+} \rightarrow \mathrm{D}^{0} \pi^{+}$

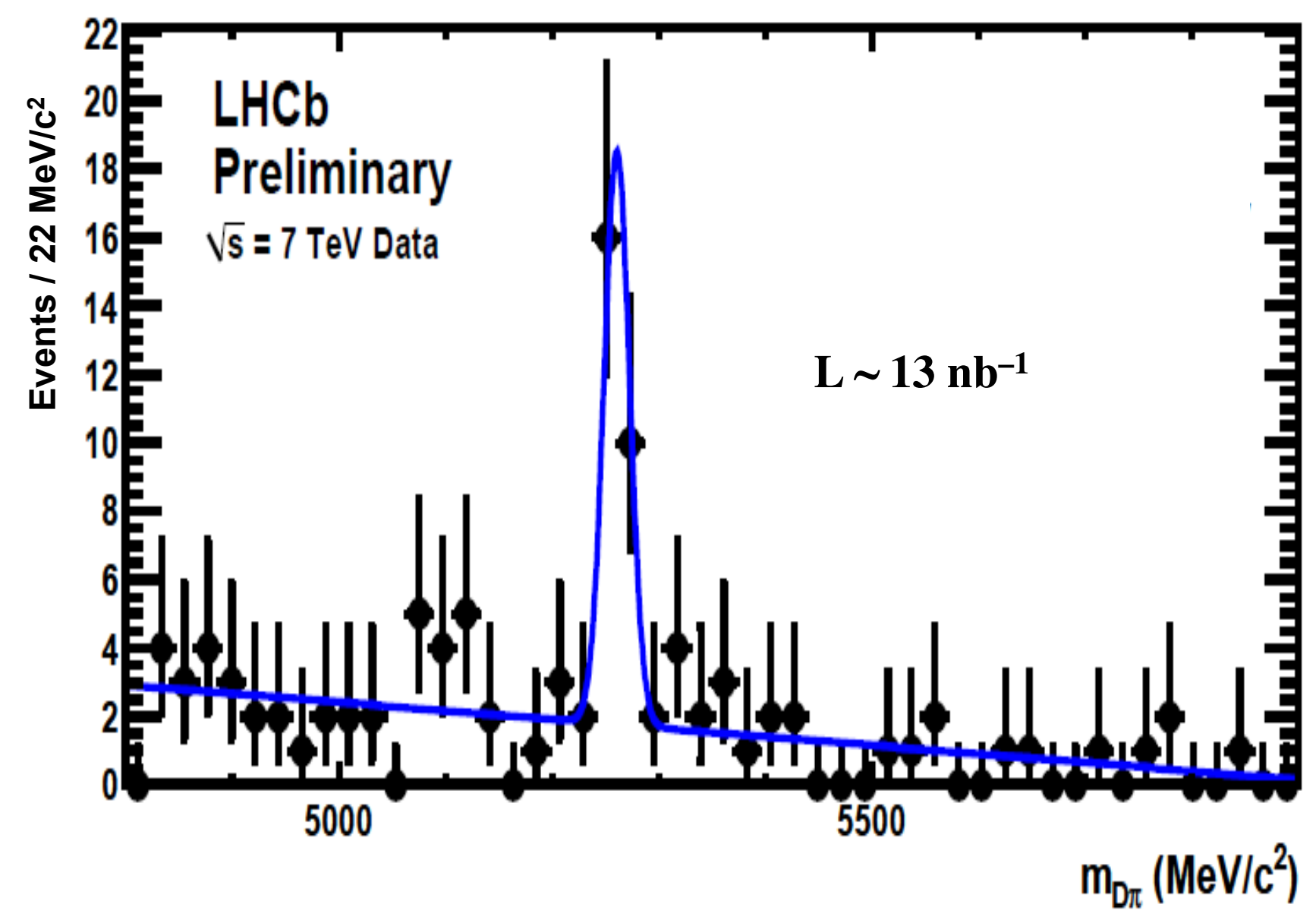




\section{$\mathrm{LHCb}$ Prospects for

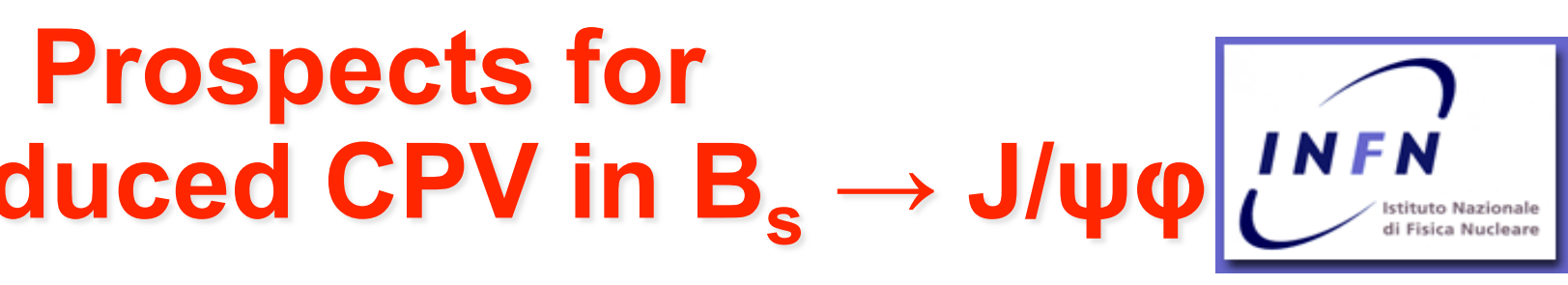

- $B_{s}$ mixing phase can be accessed with $B_{s} \rightarrow J / \Psi \varphi$ in the same way as $2 \beta$ with $\mathrm{B}^{0} \rightarrow \mathrm{J} / \psi \mathrm{K}^{0}$

- Phase $2 \beta_{\mathrm{s}}$ small in SM, hence very sensitive to NP contributions

- $2 \beta_{\mathrm{s}}=0.036 \pm 0.002 \mathrm{rad}(\mathrm{SM})$

- LHCb expectation

- With $1 \mathrm{fb}^{-1}$ and full time and angular analysis of flavour-tagged $B_{s} \rightarrow J / \Psi \varphi$ decays:

$$
\sigma\left(\varphi_{\mathrm{s}}(\mathrm{J} / \Psi \varphi)\right) \sim 0.07 \mathrm{rad}
$$

- Already during 2010 stat. for competitive sensitivity is expected

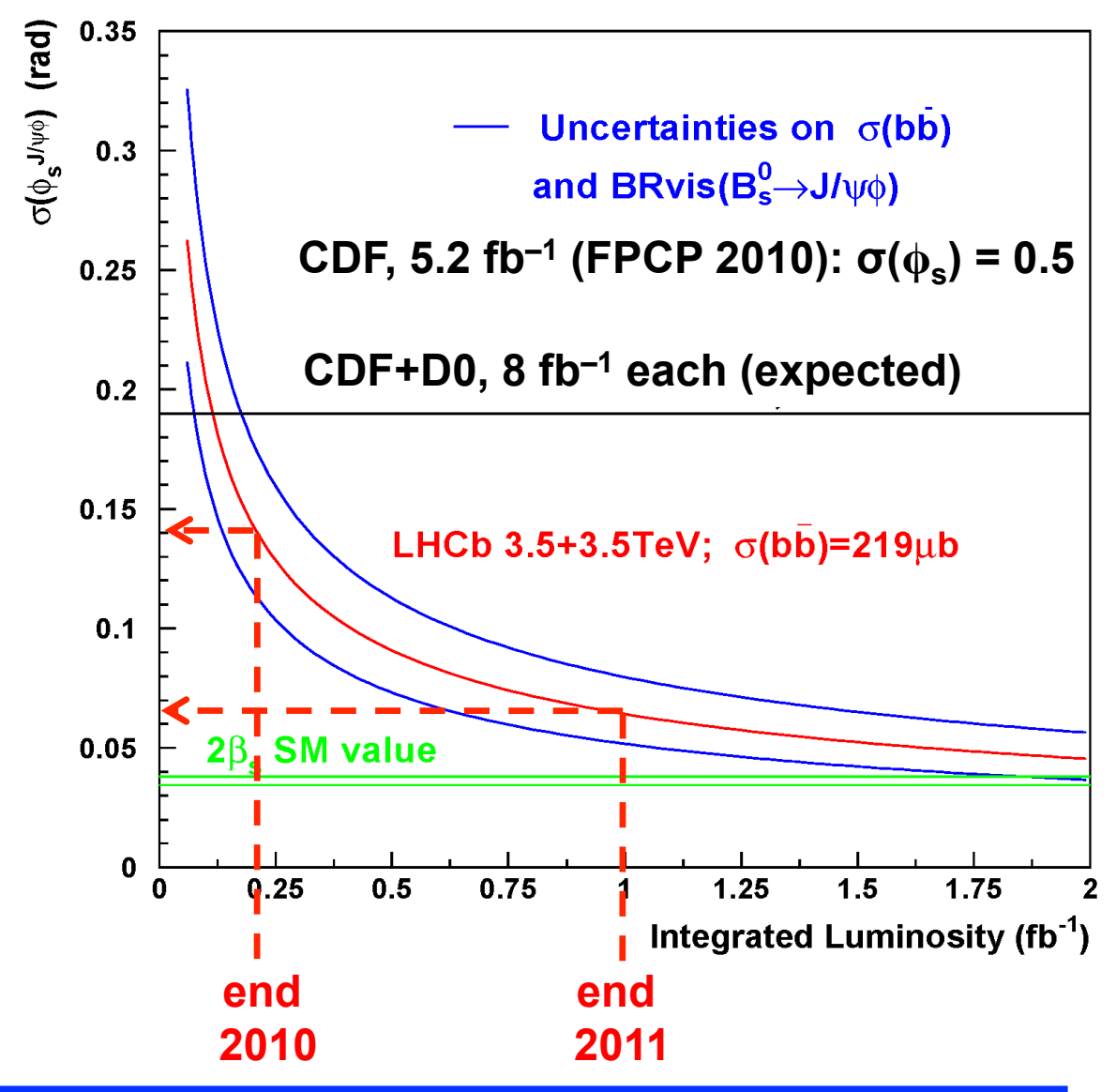


Tree-level determination of CKM angle $y$ using interference between $b \rightarrow c$ and $b \rightarrow u$ tree-level diagrams in $B_{(s)} \rightarrow D_{(s)} K$

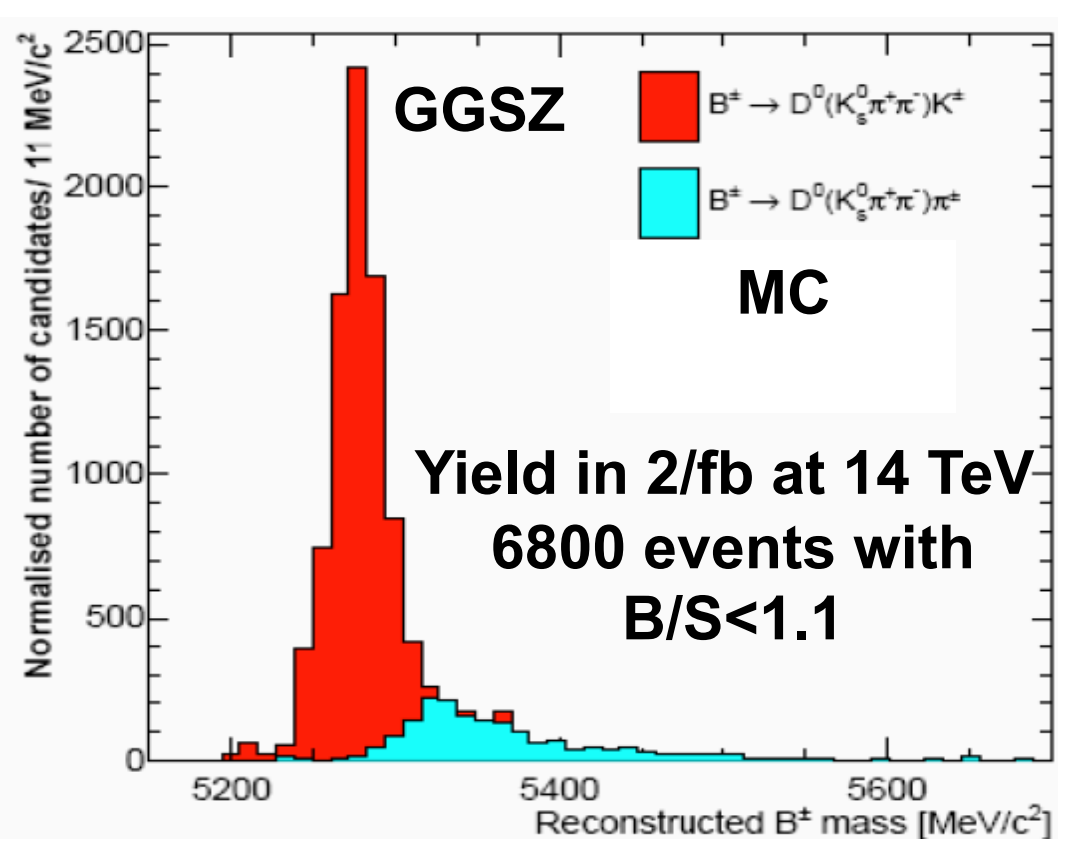

- combined precision expected with $1 \mathrm{fb}^{-1}$ at $7 \mathrm{TeV}$ :

$$
\sigma(\mathrm{Y}) \sim 7 \text { degrees }
$$

ADS/GLW modes

\begin{tabular}{|c|c|c|}
\hline Mode & MC Yield: $14 \mathrm{TeV}, 2 \mathrm{fb}^{-1}$ & MC B/S \\
\hline $\mathrm{B} \rightarrow \mathrm{D}(\mathrm{K} \pi) \mathrm{K}$ & $84 \mathrm{k}$ & 0.6 \\
\hline$B \rightarrow D_{\text {sup }}(K \pi) K$ & $1.6 \mathrm{k}$ & 0.6 \\
\hline $\mathrm{B} \rightarrow \mathrm{D}(\mathrm{K} \pi \pi \pi) \mathrm{K}$ & $53 \mathrm{k}$ & 0.2 \\
\hline $\mathrm{B} \rightarrow \mathrm{D}_{\text {sup }}(\mathrm{K} \pi \pi \pi) \mathrm{K}$ & $0.55 \mathrm{k}$ & 3.1 \\
\hline$B \rightarrow D(h h) K$ & $11.4 \mathrm{k}$ & 1.4 \\
\hline$B^{\theta} \rightarrow D(K \pi) K^{*}$ & $3.2 \mathrm{k}$ & 0.25 \\
\hline $\mathrm{B}^{\theta} \rightarrow \mathrm{D}_{\text {sup }}(\mathrm{K} \pi) \mathrm{K}^{*}$ & $0.3 \mathrm{k}$ & $<10$ \\
\hline$B^{\theta} \rightarrow D(h h) K^{*}$ & $0.4 \mathrm{k}$ & $<8$ \\
\hline 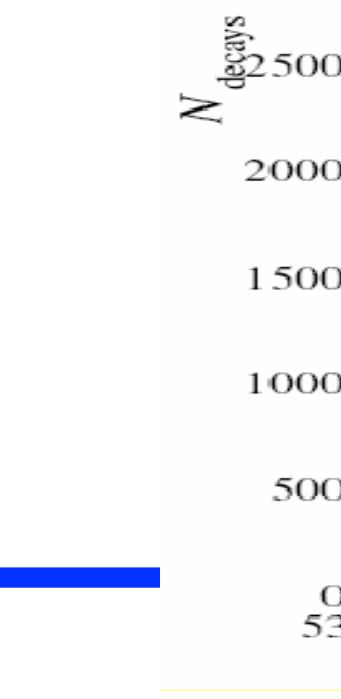 & $f \frac{\mathrm{Ma}}{\mathrm{Yi} \boldsymbol{\epsilon}}$ & $\begin{array}{l}\bullet B_{s} \rightarrow D_{s} K \\
\Delta B_{s} \rightarrow D_{s} \pi \\
\mathrm{MC} \\
\text { window } \\
\text { in 2/fb at 14 TeV } \\
00 \text { events with } \\
\text { B/S<0.5 } \\
m_{\text {inv }}(\mathrm{MeV})\end{array}$ \\
\hline
\end{tabular}


- Competitive measurements already possible with $\mathrm{L}=200 \mathrm{pb}^{-1}$

- E.g. $B_{s} \rightarrow K \pi$ charge asymmetry, relative BR's, ...

- $B_{s} \rightarrow K K$ Lifetime

- With $500 \mathrm{pb}^{-1}$ we will overcome the Bfactory stat. in the $B_{d}$ sector and measurements of time dependent $\mathrm{CP}$ asymmetries will be possible

- Maybe first measurement of $B_{s} \rightarrow K K$ time dependent CPV

- Time dep. CP asymm. sensitive to $\gamma$ and NP

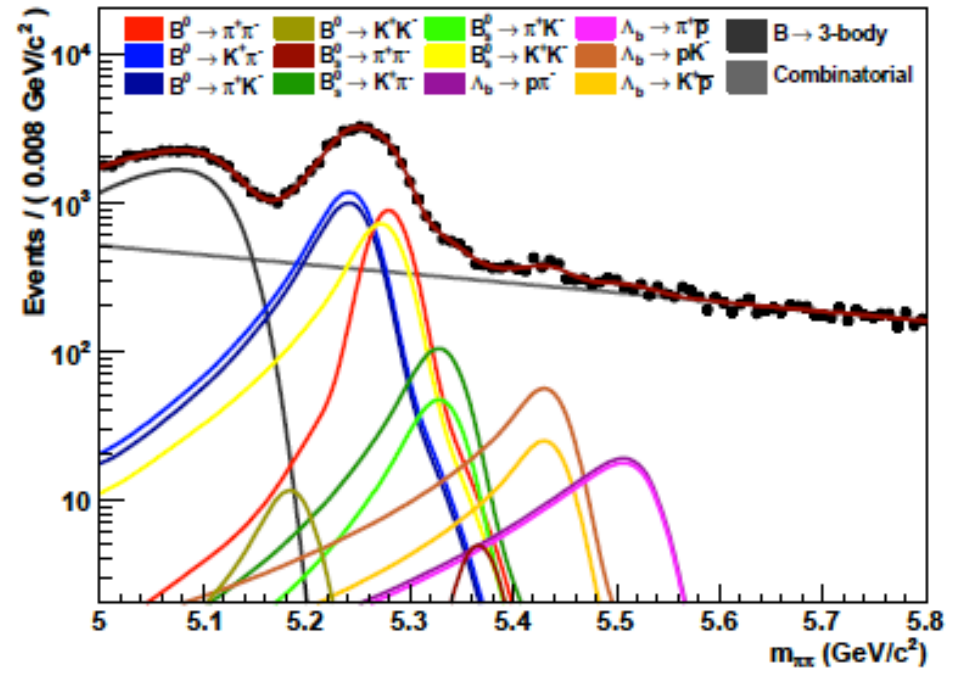

Current LHCb knowledge stat.

\begin{tabular}{|c|c|c|}
\hline $\mathcal{A}_{K+\pi}^{C P}$ & $-0.098_{-0.011}^{+0.012}$ & 0.008 \\
\hline $\mathcal{A}_{\pi+K-}^{C P}$ & $0.39 \pm 0.15 \pm 0.08$ & 0.05 \\
\hline $\mathcal{A}_{p \pi-}^{C \tilde{P}}$ & $0.03 \pm 0.17 \pm 0.05$ & 0.05 \\
\hline$A_{p K-}^{C P}$ & $0.37 \pm 0.17 \pm 0.03$ & 0.03 \\
\hline $\mathcal{A}_{\pi+\pi^{-}}^{\text {dir }}$ & $0.38 \pm 0.06$ & 0.13 \\
\hline $\mathcal{A}_{\tau^{+} \tau_{-}}^{\operatorname{mix}}$ & $-0.65 \pm 0.07$ & 0.13 \\
\hline $\operatorname{Corr}\left(\mathcal{A}_{\pi^{+} \pi^{-}}^{\text {dir }}, \mathcal{A}_{\pi^{+} \pi^{-}}^{\text {mix }}\right)$ & 0.08 & -0.03 \\
\hline $\mathcal{A}_{K^{+} K^{-}}^{\text {dir }}$ & & \\
\hline $\mathcal{A}_{K+K-}^{\operatorname{mix}}$ & Unmeasured & 0.11 \\
\hline $\operatorname{Corr}\left(\mathcal{A}_{K^{+} K^{-}}^{\text {dir }}, \mathcal{A}_{K^{+} K^{-}}^{\operatorname{mix}}\right)$ & & \\
\hline$\underline{\mathcal{B} \mathcal{R}\left(B^{0} \rightarrow \pi^{+} \pi^{-}\right)}$ & $0964+0011$ & 0.006 \\
\hline$\overline{\mathcal{B} \mathcal{R}\left(B^{0} \rightarrow K^{+} \pi^{-}\right)}$ & $0.204 \pm 0.011$ & 0.006 \\
\hline $\mathcal{B} \mathcal{R}\left(B^{0} \rightarrow K^{+} K^{-}\right)$ & 006 & 0.005 \\
\hline$\overline{\mathcal{B} \mathcal{R}\left(B^{0} \rightarrow K^{+} \pi^{-}\right)}$ & $0.020 \pm 0.008 \pm 0.006$ & 0.005 \\
\hline$f_{s} \mathcal{B} \mathcal{R}\left(B_{s}^{0} \rightarrow K^{+} K^{-}\right)$ & $0.247+0000+0.021$ & 0.006 \\
\hline$\overline{f_{d} \mathcal{B} \mathcal{R}\left(B^{0} \rightarrow K^{+} \pi^{-}\right)}$ & $0.347 \pm 0.020 \pm 0.021$ & 0.006 \\
\hline$f_{s} \mathcal{B} \mathcal{R}\left(B_{s}^{0} \rightarrow \pi^{+} K^{-}\right)$ & & \\
\hline$f_{d} \mathcal{B} \mathcal{R}\left(B^{0} \rightarrow K^{+} \pi^{-}\right)$ & $0.071 \pm 0.010 \pm 0.007$ & 0.004 \\
\hline$f_{s} \mathcal{B} \mathcal{R}\left(B_{s}^{0} \rightarrow \pi^{+} \pi^{-}\right)$ & $0007+0.004+0.005$ & בס00 \\
\hline$\overline{f_{d} \mathcal{B R}\left(B^{0} \rightarrow K^{+} \pi^{-}\right)}$ & $0.007 \pm 0.004 \pm 0.005$ & 0.002 \\
\hline$f_{\Lambda_{b}} \mathcal{B} \mathcal{R}\left(\Lambda_{b} \rightarrow p \pi^{-}\right)$ & & \\
\hline$\overline{f_{d} \mathcal{B} \mathcal{R}\left(B^{0} \rightarrow K^{+} \pi^{-}\right)}$ & 0. & 0.0016 \\
\hline$f_{\Lambda_{b}} \mathcal{B R}\left(\Lambda_{b} \rightarrow p K^{-}\right)$ & & \\
\hline$\overline{f_{d} \mathcal{B} \mathcal{R}\left(B^{0} \rightarrow K^{+} \pi^{-}\right)}$ & $0663 \pm 0.0089 \pm$ & 0.0018 \\
\hline
\end{tabular}

LHCb stat. sensitivity with $500 \mathrm{fb}^{-1}$ 
- LHCb is fully operational

- Recorded lumi so far around 14 nb-1 $^{-1}$

- Even with such a low luminosity, many interesting signals already observed

- Currently LHCb expects to collect approximately $L=0.2 \mathrm{fb}^{-1}$ in 2010 and $L=1 \mathrm{fb}^{-1}$ at the end of 2011 at $\sqrt{ }_{\mathrm{s}}=7 \mathrm{TeV}$

- Center of mass energy of $7 \mathrm{TeV}$ does not represent a major problem for 2010-2011, factor 2 in b $\bar{b}$ production cross section (value of which is still unknown)

- New Physics searches already very competitive with 2010-2011 run

- In particular using $D$ decays, measuring the $B_{s}$ mixing phase from $B_{s} \rightarrow J / \Psi \varphi, \gamma$ with tree decays from $B \rightarrow D K$ modes and with loops from $B \rightarrow$ hh modes

Waiting for more statistics......................... thank you! 